\title{
Approximation of Eigenvalues of Sturm-Liouville Problems by Using Hermite Interpolation
}

\author{
M. M. Tharwat ${ }^{1,2}$ and S. M. Al-Harbi ${ }^{3}$ \\ ${ }^{1}$ Department of Mathematics, Faculty of Science, King Abdulaziz University, Jeddah, Saudi Arabia \\ ${ }^{2}$ Department of Mathematics, Faculty of Science, Beni-Suef University, Beni Suef, Egypt \\ ${ }^{3}$ Department of Mathematics, University College, Umm Al-Qura University, P.O. Box 8140, Makkah, Saudi Arabia
}

Correspondence should be addressed to M. M. Tharwat; zahraa26@yahoo.com

Received 5 August 2013; Accepted 21 September 2013

Academic Editor: Dumitru Baleanu

Copyright (C) 2013 M. M. Tharwat and S. M. Al-Harbi. This is an open access article distributed under the Creative Commons Attribution License, which permits unrestricted use, distribution, and reproduction in any medium, provided the original work is properly cited.

Eigenvalue problems with eigenparameter appearing in the boundary conditions usually have complicated characteristic determinant where zeros cannot be explicitly computed. In this paper, we use the derivative sampling theorem "Hermite interpolations" to compute approximate values of the eigenvalues of Sturm-Liouville problems with eigenvalue parameter in one or two boundary conditions. We use recently derived estimates for the truncation and amplitude errors to compute error bounds. Also, using computable error bounds, we obtain eigenvalue enclosures. Also numerical examples, which are given at the end of the paper, give comparisons with the classical sinc method and explain that the Hermite interpolations method gives remarkably better results.

\section{Introduction}

The mathematical modeling of many practical problems in mechanics and other areas of mathematical physics requires solutions of boundary value problems (see, [1-7]) and fractional differential equations (see, [8-13]). It is well known that many topics in mathematical physics require the investigation of the eigenvalues and eigenfunctions of Sturm-Liouville type boundary value problems. The literature on computing eigenvalues of various types of Sturm-Liouville problems is little and we refer to [14-17].

Let $\sigma>0$ and let $\mathrm{PW}_{\sigma}^{2}$ be the Paley-Wiener space of all $L^{2}(\mathbb{R})$, entire functions of exponential type $\sigma$. Assume that $f(t) \in \mathrm{PW}_{\sigma}^{2} \subset \mathrm{PW}_{2 \sigma}^{2}$. Then $f(t)$ can be reconstructed via the Hermite-type sampling series as

$$
\begin{aligned}
f(t)=\sum_{n=-\infty}^{\infty}[ & f\left(\frac{n \pi}{\sigma}\right) S_{n}^{2}(t) \\
& \left.+f^{\prime}\left(\frac{n \pi}{\sigma}\right) \frac{\sin (\sigma t-n \pi)}{\sigma} S_{n}(t)\right],
\end{aligned}
$$

where $S_{n}(t)$ is the sequences of sinc functions as follows:

$$
S_{n}(t):= \begin{cases}\frac{\sin (\sigma t-n \pi)}{(\sigma t-n \pi)}, & t \neq \frac{n \pi}{\sigma}, \\ 1, & t=\frac{n \pi}{\sigma} .\end{cases}
$$

Series (1) converges absolutely and uniformly on $\mathbb{R}$, cf. [18-21]. Sometimes, series (1) is called the derivative sampling theorem. Our task is to use (1) to compute eigenvalues of Sturm-Liouville problems with eigenvalue parameter in boundary conditions numerically. This approach is a fully new technique that uses the recently obtained estimates for the truncation and amplitude errors associated with (1), cf. [22]. Both types of errors normally appear in numerical techniques that use interpolation procedures. In the following we summarize these estimates. The truncation error associated with (1) is defined to be

$$
R_{N}(f)(t):=f(t)-f_{N}(t), \quad N \in \mathbb{Z}^{+}, t \in \mathbb{R}
$$


where $f_{N}(t)$ is the truncated series as follows:

$$
\begin{aligned}
f_{N}(t)=\sum_{|n| \leq N}[ & f\left(\frac{n \pi}{\sigma}\right) S_{n}^{2}(t) \\
& \left.+f^{\prime}\left(\frac{n \pi}{\sigma}\right) \frac{\sin (\sigma t-n \pi)}{\sigma} S_{n}(t)\right] .
\end{aligned}
$$

It is proved in [22] that if $f(t) \in \mathrm{PW}_{\sigma}^{2}$ and $f(t)$ is sufficiently smooth in the sense that there exists $k \in \mathbb{Z}^{+}$such that $t^{k} f(t) \in$ $L^{2}(\mathbb{R})$, then for $t \in \mathbb{R},|t|<N \pi / \sigma$, we have

$$
\begin{aligned}
\left|R_{N}(f)(t)\right| & \\
\leq & T_{N, k, \sigma}(t) \\
:= & \frac{\xi_{k, \sigma} E_{k}|\sin \sigma t|^{2}}{\sqrt{3}(N+1)^{k}}\left(\frac{1}{(N \pi-\sigma t)^{3 / 2}}+\frac{1}{(N \pi+\sigma t)^{3 / 2}}\right) \\
& +\frac{\xi_{k, \sigma}\left(\sigma E_{k}+k E_{k-1}\right)|\sin \sigma t|^{2}}{\sigma(N+1)^{k}} \\
& \times\left(\frac{1}{\sqrt{N \pi-\sigma t}}+\frac{1}{\sqrt{N \pi+\sigma t}}\right)
\end{aligned}
$$

where the constants $E_{k}$ and $\xi_{k, \sigma}$ are given by

$$
E_{k}:=\sqrt{\int_{-\infty}^{\infty}\left|t^{k} f(t)\right|^{2} d t}, \quad \xi_{k, \sigma}:=\frac{\sigma^{k+1 / 2}}{\pi^{k+1} \sqrt{1-4^{-k}}} .
$$

The amplitude error occurs when approximate samples are used instead of the exact ones, which we cannot compute. It is defined to be

$$
\begin{aligned}
& \mathscr{A}(\varepsilon, f)(t) \\
& =\sum_{n=-\infty}^{\infty}\left[\left\{f\left(\frac{n \pi}{\sigma}\right)-\tilde{f}\left(\frac{n \pi}{\sigma}\right)\right\} S_{n}^{2}(t)\right. \\
& \left.+\left\{f^{\prime}\left(\frac{n \pi}{\sigma}\right)-\widetilde{f^{\prime}}\left(\frac{n \pi}{\sigma}\right)\right\} \frac{\sin (\sigma t-n \pi)}{\sigma} S_{n}(t)\right], \\
& t \in \mathbb{R},
\end{aligned}
$$

where $\widetilde{f}(n \pi / \sigma)$ and $\widetilde{f^{\prime}}(n \pi / \sigma)$ are approximate samples of $f(n \pi / \sigma)$ and $f^{\prime}(n \pi / \sigma)$, respectively. Let us assume that the differences $\varepsilon_{n}:=f(n \pi / \sigma)-\tilde{f}(n \pi / \sigma), \varepsilon_{n}^{\prime}:=f^{\prime}(n \pi / \sigma)-$ $\widetilde{f^{\prime}}(n \pi / \sigma)$, and $n \in \mathbb{Z}$ are bounded by a positive number $\varepsilon$, that is, $\left|\varepsilon_{n}\right|,\left|\varepsilon_{n}^{\prime}\right| \leq \varepsilon$. If $f(t) \in \mathrm{PW}_{\sigma}^{2}$ satisfies the natural decay conditions

$$
\begin{gathered}
\left|\varepsilon_{n}\right| \leq\left|f\left(\frac{n \pi}{\sigma}\right)\right|, \quad\left|\varepsilon_{n}^{\prime}\right| \leq\left|f^{\prime}\left(\frac{n \pi}{\sigma}\right)\right|, \\
|f(t)| \leq \frac{M_{f}}{|t|^{\nu+1}}, \quad t \in \mathbb{R}-\{0\},
\end{gathered}
$$

$0<v \leq 1$, then for $0<\varepsilon \leq \min \{\pi / \sigma, \sigma / \pi, 1 / \sqrt{e}\}$, we have, [22],

$$
\begin{aligned}
\|\mathscr{A}(\varepsilon, f)\|_{\infty} & \\
\leq \frac{4 e^{1 / 4}}{\sigma(\nu+1)}\{ & \sqrt{3} e(1+\sigma)+\left(\left(\frac{\pi}{\sigma}\right) A+M_{f}\right) \rho(\varepsilon) \\
& \left.+(\sigma+2+\log (2)) M_{f}\right\} \varepsilon \log \left(\frac{1}{\varepsilon}\right),
\end{aligned}
$$

where

$$
\begin{gathered}
A:=\frac{3 \sigma}{\pi}\left(|f(0)|+M_{f}\left(\frac{\sigma}{\pi}\right)^{\nu}\right), \\
\rho(\varepsilon):=\gamma+10 \log \left(\frac{1}{\varepsilon}\right),
\end{gathered}
$$

and $\gamma:=\lim _{n \rightarrow \infty}\left[\sum_{k=1}^{n} 1 / k-\log n\right] \cong 0.577216$ is the EulerMascheroni constant.

The classical [23] sampling theorem of Whittaker, Kotelnikov, and Shannon (WKS) for $f \in \mathrm{PW}_{\sigma}^{2}$ is the series representation as follows:

$$
f(t)=\sum_{n=-\infty}^{\infty} f\left(\frac{n \pi}{\sigma}\right) S_{n}(t), \quad t \in \mathbb{R},
$$

where the convergence is absolute and uniform on $\mathbb{R}$ and it is uniform on compact sets of $\mathbb{C}$ cf. [23-25]. Series (12), which is of Lagrange interpolation type, has been used to compute eigenvalues of second-order eigenvalue problems, see for example, [17, 26-29]. The use of (12) in numerical analysis is known as the sinc method established by Stenger et al., cf. [30-32]. The aim of this paper is to investigate the possibilities of using Hermite interpolations rather than Lagrange interpolations, to compute the eigenvalues numerically. Notice that, due to Paley-Wiener's theorem [33] $f \in$ $\mathrm{PW}_{\sigma}^{2}$ if and only if there is $g(\cdot) \in L^{2}(-\sigma, \sigma)$ such that

$$
f(t)=\frac{1}{\sqrt{2 \pi}} \int_{-\sigma}^{\sigma} g(x) e^{i x t} d x
$$

Therefore, $f^{\prime}(t) \in \mathrm{PW}_{\sigma}^{2}$, that is, $f^{\prime}(t)$ also has an expansion of the form (12). However, $f^{\prime}(t)$ can also be obtained by termby-term differentiation formula of (12) as follows:

$$
f^{\prime}(t)=\sum_{n=-\infty}^{\infty} f\left(\frac{n \pi}{\sigma}\right) S_{n}^{\prime}(t)
$$

see [23, page 52] for convergence. Thus, the use of Hermite interpolations will not cost any additional computational efforts since the samples $f(n \pi / \sigma)$ will be used to compute both $f(t)$ and $f^{\prime}(t)$ according to (12) and (14), respectively.

Now, we consider the following differential equations:

$$
\begin{array}{r}
\ell(y):=-y^{\prime \prime}(x, \mu)+q(x) y(x, \mu)=\mu^{2} y(x, \mu), \\
x \in[0,1],
\end{array}
$$


with the following boundary conditions:

$$
\begin{aligned}
& a_{1} y(0, \mu)+a_{2} y^{\prime}(0, \mu)=\mu^{2}\left(a_{1}^{\prime} y(0, \mu)+a_{2}^{\prime} y^{\prime}(0, \mu)\right), \\
& b_{1} y(1, \mu)+b_{2} y^{\prime}(1, \mu)=\mu^{2}\left(b_{1}^{\prime} y(1, \mu)+b_{2}^{\prime} y^{\prime}(1, \mu)\right)
\end{aligned}
$$

where $\mu$ is a complex spectral parameter, $q(\cdot)$ is assumed to be real valued and continuous on $[0,1]$, and $a_{i}, b_{i}, a_{i}^{\prime}, b_{i}^{\prime} \in \mathbb{R}$, $i=0,1$ satisfying

$$
\begin{aligned}
& \left(\left(a_{1}^{\prime}, a_{2}^{\prime}\right)=(0,0) \text { or } a_{1} a_{2}^{\prime}-a_{1}^{\prime} a_{2}>0\right) \\
& \left(\left(b_{1}^{\prime}, b_{2}^{\prime}\right)=(0,0) \text { or } b_{1}^{\prime} b_{2}-b_{1} b_{2}^{\prime}>0\right)
\end{aligned}
$$

The eigenvalue problem (15)-(17) will be denoted by $\Pi\left(q, a, b, a^{\prime}, b^{\prime}\right)$ when $\left(a_{1}^{\prime}, a_{2}^{\prime}\right) \neq(0,0) \neq\left(b_{1}^{\prime}, b_{2}^{\prime}\right)$. It is a SturmLiouville problem when the eigenparameter $\mu$ appears linearly in both boundary conditions. The classical problem when $a_{1}^{\prime}=a_{2}^{\prime}=b_{1}^{\prime}=b_{2}^{\prime}=0$, which we denote by $\Pi(q, a, b, 0$, 0 ) has a countable set of real and simple eigenvalues with $\infty$ as the only possible limit point, [34, 35]. In [14], the authors used Hermite-type sampling series (1) to compute the eigenvalues of problem $\Pi(q, a, b, 0,0)$ numerically. In [36], see also [37], Annaby and Tharwat proved that $\Pi\left(q, a, b, a^{\prime}, b^{\prime}\right)$ has a denumerable set of real and simple eigenvalues with $\infty$ as the limit point using techniques similar of those established in [38-40], where also sampling theorems have been established. Similar results are established in [38] for the problem when the eigenparameter appears in one condition, that is, when $a_{1}^{\prime}=a_{2}^{\prime}=0,\left(b_{1}^{\prime}, b_{2}^{\prime}\right) \neq(0,0)$ or equivalently when $\left(a_{1}^{\prime}, a_{2}^{\prime}\right) \neq(0,0)$ and $b_{1}^{\prime}=b_{2}^{\prime}=0$. These problems will be denoted by $\Pi\left(q, a, b, 0, b^{\prime}\right), \Pi\left(q, a, b, a^{\prime}, 0\right)$, respectively. The aim of the present work is to compute the eigenvalues of $\Pi\left(q, a, b, a^{\prime}, b^{\prime}\right), \Pi\left(q, a, b, 0, b^{\prime}\right)$, and $\Pi\left(q, a, b, a^{\prime}, 0\right)$ numerically by the Hermite interpolations with an error analysis. This method is based on sampling theorem, Hermite interpolations, but applied to regularized functions. Hence, avoiding any (multiple) integration and keeping the number of terms in the Cardinal series manageable. It has been demonstrated that the method is capable of delivering higher order estimates of the eigenvalues at a very low cost, see [4143]. In Sections 2 and 3 we derive the Hermite interpolation technique to compute the eigenvalues of $\Pi\left(q, a, b, a^{\prime}, b^{\prime}\right)$ and $\Pi\left(q, a, b, 0, b^{\prime}\right)$ with error estimates, respectively. The last section involves some illustrative examples.

\section{Treatment of $\Pi\left(q, a, b, a^{\prime}, b^{\prime}\right)$}

In this section, we derive approximate values of the eigenvalues of $\Pi\left(q, a, b, a^{\prime}, b^{\prime}\right)$. Let $y(\cdot, \mu)$ denote the solution of (15) satisfying the following initial conditions:

$$
y(0, \mu)=a_{2}-a_{2}^{\prime} \mu^{2}, \quad y^{\prime}(0, \mu)=a_{1}^{\prime} \mu^{2}-a_{1} .
$$

Thus, $y(\cdot, \mu)$ satisfies the boundary condition (16). The eigenvalues of the problem $\Pi\left(q, a, b, a^{\prime}, b^{\prime}\right)$ are the zeros of the function as follows:

$$
\Delta(\mu):=\left(b_{1}^{\prime} \mu^{2}-b_{1}\right) y(1, \mu)+\left(b_{2}^{\prime} \mu^{2}-b_{2}\right) y^{\prime}(1, \mu) .
$$

These zeros are real and simple. The function $\Delta(\mu)$ is an entire function of $\mu$. We aim to approximate $\Delta(\mu)$ and hence its zeros, that is, the eigenvalues by the use of the Hermite Interpolation. The idea is to split $\Delta(\mu)$ into two parts, one is known and the other is unknown, but lies in a PaleyWiener space. Then we approximate the unknown part to get the approximate $\Delta(\mu)$ and then compute the approximate zeros. Using the method of variation of constants, the solution $y(x, \mu)$ satisfies Volterra integral equation as follows:

$$
\begin{aligned}
y(x, \mu)= & \left(a_{2}-a_{2}^{\prime} \mu^{2}\right) \cos \mu x \\
& -\left(a_{1}-a_{1}^{\prime} \mu^{2}\right) \frac{\sin \mu x}{\mu}+T[y](x, \mu),
\end{aligned}
$$

where $T$ is the Volterra operator defined by

$$
T[y](x, \mu)=\int_{0}^{x} \frac{\sin \mu(x-t)}{\mu} q(t) y(t, \mu) d t .
$$

Differentiating (21), we get

$$
\begin{aligned}
y^{\prime}(x, \mu)= & \left(a_{2}^{\prime} \mu^{2}-a_{2}\right) \mu \sin \mu x \\
& +\left(a_{1}^{\prime} \mu^{2}-a_{1}\right) \cos \mu x+\widetilde{T}[y](x, \mu),
\end{aligned}
$$

where $\widetilde{T}$ is the Volterra operator

$$
\widetilde{T}[y](x, \mu)=\int_{0}^{x} \cos \mu(x-t) q(t) y(t, \mu) d t .
$$

Define $f(\cdot, \mu)$ and $g(\cdot, \mu)$ to be

$$
f(x, \mu):=T[y](x, \mu), \quad g(x, \mu):=\widetilde{T}[y](x, \mu) .
$$

In the following, we will make use of the estimates [44] as follows:

$$
|\cos z| \leq e^{|\Im z|}, \quad\left|\frac{\sin z}{z}\right| \leq \frac{c_{0}}{1+|z|} e^{|\Im z|},
$$

where $c_{0}$ is some constant (we may take $c_{0} \simeq 1.72$ ). For convenience, we define the constants by

$$
\begin{gathered}
\tau:=\int_{0}^{1}|q(t)| d t, \quad c_{1}:=\left|a_{2}\right|+c_{0}\left|a_{1}\right|, \\
c_{2}:=\left|a_{2}^{\prime}\right|+c_{0}\left|a_{1}^{\prime}\right|, \quad c_{3}:=c_{0} \tau, \\
c_{4}:=\exp c_{3}, \quad c_{5}:=\max \left\{c_{1}, c_{2},\left|b_{1}\right|+\left|b_{2}\right| \tau,\left|b_{1}^{\prime}\right|+\left|b_{2}^{\prime}\right| \tau\right\} .
\end{gathered}
$$

From (21) and (25), we get

$$
\begin{gathered}
f(x, \mu) \\
=\int_{0}^{x} \frac{\sin \mu(x-t)}{\mu} q(t)\left[\left(a_{2}-a_{2}^{\prime} \mu^{2}\right) \cos \mu t\right. \\
\left.\quad-\left(a_{1}-a_{1}^{\prime} \mu^{2}\right) \frac{\sin \mu t}{\mu}\right] d t \\
+\int_{0}^{x} \frac{\sin \mu(x-t)}{\mu} q(t) f(t, \mu) d t .
\end{gathered}
$$


Lemma 1. For $0 \leq x \leq 1, \mu \in \mathbb{C}$, the following estimates hold:

$$
\begin{aligned}
& f(x, \mu) \leq \frac{c_{3} c_{4}\left(c_{1}+c_{2}|\mu|^{2}\right)}{1+|\mu|} e^{|\Im \mu| x}, \\
& g(x, \mu) \leq \frac{\tau c_{3} c_{4}\left(c_{1}+c_{2}|\mu|^{2}\right)}{1+|\mu|} e^{|\Im \mu| x} .
\end{aligned}
$$

Proof. We divide $f(\cdot, \mu)$ into two parts $f_{1}(\cdot, \mu)$ and $f_{2}(\cdot, \mu)$ and estimate each of them. Indeed, for $x \in[0,1]$ and $\mu \in \mathbb{C}$ we have

$$
\begin{aligned}
& \left|f_{1}(x, \mu)\right| \\
& =\mid \int_{0}^{x} \frac{\sin \mu(x-t)}{\mu} q(t)\left[\left(a_{2}-a_{2}^{\prime} \mu^{2}\right) \cos \mu t\right. \\
& \left.-\left(a_{1}-a_{1}^{\prime} \mu^{2}\right) \frac{\sin \mu t}{\mu}\right] d t \\
& \leq e^{|\Im \mu| x} \int_{0}^{x}|q(t)| \frac{c_{0}(x-t)}{1+|\mu|(x-t)} \\
& \times\left[\left|a_{2}\right|+\left|a_{2}^{\prime}\right||\mu|^{2}+\left(\left|a_{1}\right|+\left|a_{1}^{\prime}\right||\mu|^{2}\right)\right. \\
& \left.\times \frac{c_{0} t}{1+|\mu| t}\right] d t \\
& \leq e^{|\Im \mu| x} \frac{c_{0} x}{1+|\mu| x} \int_{0}^{x}|q(t)|\left[\left|a_{2}\right|+\left|a_{2}^{\prime}\right||\mu|^{2}\right. \\
& \left.+\left(\left|a_{1}\right|+\left|a_{1}^{\prime}\right||\mu|^{2}\right) c_{0} t\right] d t \\
& \leq e^{|\Im \mu| x} \frac{c_{0}}{1+|\mu|} \int_{0}^{1}|q(t)|\left[\left|a_{2}\right|+\left|a_{2}^{\prime}\right||\mu|^{2}\right. \\
& \left.+\left(\left|a_{1}\right|+\left|a_{1}^{\prime}\right||\mu|^{2}\right) c_{0} t\right] d t
\end{aligned}
$$

Moreover, $0 \leq x \leq 1, \mu \in \mathbb{C}$,

$$
\begin{aligned}
\left|f_{2}(x, \mu)\right|= & \left|\int_{0}^{x} \frac{\sin \mu(x-t)}{\mu} q(t) f(t, \mu) d t\right| \\
\leq & \int_{0}^{x} \frac{c_{0}(x-t)}{1+|\mu|(x-t)} e^{|\Im \mu|(x-t)} \\
& \times|q(t)||f(t, \mu)| d t \\
\leq & c_{0} e^{|\Im \mu| x} \int_{0}^{x} e^{-|\Im \mu| t}|q(t)||f(t, \mu)| d t .
\end{aligned}
$$

Combining (31) and (32), we obtain $0 \leq x \leq 1, \mu \in \mathbb{C}$,

$$
\begin{aligned}
& |f(x, \mu)| \\
& \leq e^{|\Im \mu| x} \frac{c_{0}}{1+|\mu|} \int_{0}^{1}|q(t)|\left[\left|a_{2}\right|+\left|a_{2}^{\prime}\right||\mu|^{2}\right. \\
& \left.+\left(\left|a_{1}\right|+\left|a_{1}^{\prime}\right||\mu|^{2}\right) c_{0} t\right] d t \\
& +c_{0} e^{|\Im \mu| x} \int_{0}^{x} e^{-|\Im \mu| t}|q(t)||f(t, \mu)| d t .
\end{aligned}
$$

Applying Gronwall's inequality, cf. for example, [34, page 51], yields $\mu \in \mathbb{C}$,

$$
\begin{aligned}
& e^{-|\Im \mu| x}|f(x, \mu)| \\
& \leq\left[\frac { c _ { 0 } } { 1 + | \mu | } \int _ { 0 } ^ { 1 } | q ( t ) | \left[\left|a_{2}\right|+\left|a_{2}^{\prime}\right||\mu|^{2}\right.\right. \\
& \left.\left.\quad+\left(\left|a_{1}\right|+\left|a_{1}^{\prime}\right||\mu|^{2}\right) c_{0} t\right] d t\right] \\
& \quad \times \exp \left(c_{0} \int_{0}^{x}|q(t)| d t\right) \\
& \leq\left[\frac { c _ { 0 } } { 1 + | \mu | } \int _ { 0 } ^ { 1 } | q ( t ) | \left[\left|a_{2}\right|+\left|a_{2}^{\prime}\right||\mu|^{2}\right.\right. \\
& \left.\left.\quad+\left(\left|a_{1}\right|+\left|a_{1}^{\prime}\right||\mu|^{2}\right) c_{0} t\right] d t\right] \\
& \quad \times \exp \left(c_{0} \int_{0}^{1}|q(t)| d t\right),
\end{aligned}
$$

from which we get

$$
\begin{aligned}
& |f(x, \mu)| \\
& \leq e^{|\Im \mu| x}\left[\frac{c_{0}\left[\left|a_{2}\right|+\left|a_{2}^{\prime}\right||\mu|^{2}+\left(\left|a_{1}\right|+\left|a_{1}^{\prime}\right||\mu|^{2}\right) c_{0}\right]}{1+|\mu|}\right. \\
& \left.\times \int_{0}^{1}|q(t)| d t\right] \exp \left(c_{0} \int_{0}^{1}|q(t)| d t\right) \\
& =\frac{c_{3} c_{4}\left(c_{1}+c_{2}|\mu|^{2}\right)}{1+|\mu|} e^{|\Im \mu| x} .
\end{aligned}
$$

Then from (25) and (29), we obtain the estimate (30).

Now we split $\Delta(\mu)$ into two parts via

$$
\Delta(\mu)=\mathscr{G}(\mu)+\mathcal{S}(\mu),
$$

where $\mathscr{G}(\mu)$ is known part

$\mathscr{G}(\mu)$

$$
\begin{aligned}
= & \left(b_{1}^{\prime} \mu^{2}-b_{1}\right)\left[\left(a_{2}-a_{2}^{\prime} \mu^{2}\right) \cos \mu-\left(a_{1}-a_{1}^{\prime} \mu^{2}\right) \frac{\sin \mu}{\mu}\right] \\
& +\left(b_{2}^{\prime} \mu^{2}-b_{2}\right)\left[\left(a_{2}^{\prime} \mu^{2}-a_{2}\right) \mu \sin \mu+\left(a_{1}^{\prime} \mu^{2}-a_{1}\right) \cos \mu\right],
\end{aligned}
$$


and $\delta(\mu)$ is unknown part

$$
\mathcal{S}(\mu)=\left(b_{1}^{\prime} \mu^{2}-b_{1}\right) f(1, \mu)+\left(b_{2}^{\prime} \mu^{2}-b_{2}\right) g(1, \mu) .
$$

Then, from Lemma 1, we have the following lemma.

Lemma 2. The function $\delta(\mu)$ is entire in $\mu$ and the following estimate holds:

$$
|\mathcal{S}(\mu)| \leq \frac{c_{3} c_{4} c_{5}\left(1+|\mu|^{2}\right)^{2}}{1+|\mu|} e^{|\Im \mu|}
$$

Proof. Since

$$
\begin{aligned}
& \mathcal{S}(\mu) \\
& \quad \leq\left(\left|b_{1}^{\prime}\right||\mu|^{2}+\left|b_{1}\right|\right)|f(1, \mu)|+\left(\left|b_{2}^{\prime}\right||\mu|^{2}+\left|b_{2}\right|\right)|g(1, \mu)|,
\end{aligned}
$$

then from (29) and (30) we get (39).

The analyticity of $\mathcal{S}(\mu)$ and estimate (39) are not adequate to prove that $\delta(\mu)$ lies in a Paley-Wiener space. To solve this problem, we will multiply $\mathcal{S}(\mu)$ by a regularization factor. Let $\theta \in(0,1)$ and let $m \in \mathbb{Z}^{+}, m>4$ be fixed. Let $\mathscr{F}_{\theta, m}(\mu)$ be the function

$$
\mathscr{F}_{\theta, m}(\mu):=\left(\frac{\sin \theta \mu}{\theta \mu}\right)^{m} \mathcal{S}(\mu), \quad \mu \in \mathbb{C}
$$

More specifications on $m, \theta$ will be given later on. Then we have the next lemma.

Lemma 3. $\mathscr{F}_{\theta, m}(\mu)$ is an entire function of $\mu$ which satisfies the estimates

$$
\left|\mathscr{F}_{\theta, m}(\mu)\right| \leq \frac{c_{3} c_{4} c_{5} c_{0}^{m}\left(1+|\mu|^{2}\right)^{2}}{(1+\theta|\mu|)^{m+1}} e^{|\Im \mu|(1+m \theta)} .
$$

Moreover, $\mu^{m-4} \mathscr{F}_{\theta, m}(\mu) \in L^{2}(\mathbb{R})$ and

$$
E_{m-4}\left(\mathscr{F}_{\theta, m}\right)=\sqrt{\int_{-\infty}^{\infty}\left|\mu^{m-4} \mathscr{F}_{\theta, m}(\mu)\right|^{2} d \mu} \leq \sqrt{2} c_{3} c_{4} c_{5} c_{0}^{m} v_{0}
$$

where

$$
\begin{aligned}
& v_{0} \\
& \qquad=(( \\
& \left.\quad \times\left(280 \theta^{4} \Gamma[2 m-7]+20 \theta^{2} \Gamma[2 m-5]+\Gamma[2 m-3]\right)\right) \\
& \left.\quad \times\left(m\left(4 m^{2}-1\right) \Gamma[2 m+2] \theta^{2 m+1}\right)^{-1}\right)^{1 / 2} .
\end{aligned}
$$

Proof. Since $\mathcal{S}(\mu)$ is entire, then also $\mathscr{F}_{\theta, m}(\mu)$ is entire in $\mu$. Combining the estimates $|\sin z / z| \leq\left(c_{0} /(1+|z|)\right) e^{|\Im z|}$ and (39), we obtain

$$
\begin{aligned}
\left|\mathscr{F}_{\theta, m}(\mu)\right| \leq & \left(\frac{c_{0}}{1+\theta|\mu|}\right)^{m} e^{|\Im \mu| m \theta} \\
& \cdot \frac{c_{3} c_{4} c_{5}\left(1+|\mu|^{2}\right)^{2}}{1+|\mu|} e^{|\Im \mu|}, \quad \mu \in \mathbb{C},
\end{aligned}
$$

leading to (42). Therefore, we get

$$
\left|\mu^{m-4} \mathscr{F}_{\theta, m}(\mu)\right| \leq \frac{c_{3} c_{4} c_{5} c_{0}^{m}|\mu|^{m-4}\left(1+|\mu|^{2}\right)^{2}}{(1+\theta|\mu|)^{m+1}}, \quad \mu \in \mathbb{R} .
$$

That is, $\mu^{m-4} \mathscr{F}_{\theta, m}(\mu) \in L^{2}(\mathbb{R})$. Moreover, we get

$$
\begin{aligned}
& \int_{-\infty}^{\infty}\left|\mu^{m-4} \mathscr{F}_{\theta, m}(\mu)\right|^{2} d \mu \\
& \leq c_{3}^{2} c_{4}^{2} c_{5}^{2} c_{0}^{2 m} \\
& \quad \times \int_{-\infty}^{\infty} \frac{|\mu|^{2 m-8}\left(1+|\mu|^{2}\right)^{4}}{(1+\theta|\mu|)^{2 m+2}} d \mu=2 c_{3}^{2} c_{4}^{2} c_{5}^{2} c_{0}^{2 m} v_{0}^{2} .
\end{aligned}
$$

What we have just proved is that $\mathscr{F}_{\theta, m}(\mu)$ belongs to the Paley-Wiener space $\mathrm{PW}_{\sigma}^{2}$ with $\sigma=1+m \theta$. Since $\mathscr{F}_{\theta, m}(\mu) \epsilon$ $\mathrm{PW}_{\sigma}^{2} \subset \mathrm{PW}_{2 \sigma}^{2}$, then we can reconstruct the functions $\mathscr{F}_{\theta, m}(\mu)$ via the following sampling formula:

$$
\begin{aligned}
\mathscr{F}_{\theta, m}(\mu)=\sum_{n=-\infty}^{\infty}\left[\mathscr{F}_{\theta, m}\left(\frac{n \pi}{\sigma}\right) S_{n}^{2}(\mu)\right. \\
\\
\left.\quad+\mathscr{F}_{\theta, m}^{\prime}\left(\frac{n \pi}{\sigma}\right) \frac{\sin (\sigma \mu-n \pi)}{\sigma} S_{n}(\mu)\right] .
\end{aligned}
$$

Let $N \in \mathbb{Z}^{+}, N>m$ and approximate $\mathscr{F}_{\theta, m}(\mu)$ by its truncated series $\mathscr{F}_{\theta, m, N}(\mu)$, where

$$
\begin{aligned}
\mathscr{F}_{\theta, m, N}(\mu):=\sum_{n=-N}^{N}\left[\mathscr{F}_{\theta, m}\left(\frac{n \pi}{\sigma}\right) S_{n}^{2}(\mu)\right. \\
\\
\left.\quad+\mathscr{F}_{\theta, m}^{\prime}\left(\frac{n \pi}{\sigma}\right) \frac{\sin (\sigma \mu-n \pi)}{\sigma} S_{n}(\mu)\right] .
\end{aligned}
$$

Since all eigenvalues are real, then from now on we restrict ourselves to $\mu \in \mathbb{R}$. Since $\mu^{m-4} \mathscr{F}_{\theta, m}(\mu) \in L^{2}(\mathbb{R})$, the truncation error, cf. (5), is given for $|\mu|<N \pi / \sigma$ by

$$
\left|\mathscr{F}_{\theta, m}(\mu)-\mathscr{F}_{\theta, m, N}(\mu)\right| \leq T_{N, m-4, \sigma}(\mu),
$$


where

$$
\begin{aligned}
T_{N, m-4, \sigma}(\mu) & \\
:= & \frac{\xi_{m-4, \sigma} E_{m-4}|\sin \sigma \mu|^{2}}{\sqrt{3}(N+1)^{m-4}}\left(\frac{1}{(N \pi-\sigma \mu)^{3 / 2}}+\frac{1}{(N \pi+\sigma \mu)^{3 / 2}}\right) \\
& +\frac{\xi_{m-4, \sigma}\left(\sigma E_{m-4}+(m-4) E_{m-5}\right)|\sin \sigma \mu|^{2}}{\sigma(N+1)^{m-4}} \\
& \times\left(\frac{1}{\sqrt{N \pi-\sigma \mu}}+\frac{1}{\sqrt{N \pi+\sigma \mu}}\right) .
\end{aligned}
$$

The samples $\left\{\mathscr{F}_{\theta, m}(n \pi / \sigma)\right\}_{n=-N}^{N}$ and $\left\{\mathscr{F}_{\theta, m}^{\prime}(n \pi / \sigma)\right\}_{n=-N}^{N}$, in general, are not known explicitly. So we approximate them by solving numerically $8 N+4$ initial value problems at the nodes $\{n \pi / \sigma\}_{n=-N}^{N}$. Let $\left\{\widetilde{\mathscr{F}}_{\theta, m}(n \pi / \sigma)\right\}_{n=-N}^{N}$ and let $\left\{\widetilde{\mathscr{F}}_{\theta, m}^{\prime}(n \pi / \sigma)\right\}_{n=-N}^{N}$ be the approximations of the samples of $\left\{\mathscr{F}_{\theta, m}(n \pi / \sigma)\right\}_{n=-N}^{N}$ and $\left\{\mathscr{F}_{\theta, m}^{\prime}(n \pi / \sigma)\right\}_{n=-N}^{N}$, respectively. Now we define $\widetilde{\mathscr{F}}_{\theta, m, N}(\mu)$, which approximates $\mathscr{F}_{\theta, m, N}(\mu)$ as

$$
\begin{array}{r}
\widetilde{\mathscr{F}}_{\theta, m, N}(\mu):=\sum_{n=-N}^{N}\left[\widetilde{\mathscr{F}}_{\theta, m}\left(\frac{n \pi}{\sigma}\right) S_{n}^{2}(\mu)\right. \\
\left.+\widetilde{\mathscr{F}}_{\theta, m}^{\prime}\left(\frac{n \pi}{\sigma}\right) \frac{\sin (\sigma \mu-n \pi)}{\sigma} S_{n}(\mu)\right], \\
N>m .
\end{array}
$$

Using standard methods for solving initial problems, we may assume that for $|n|<N$,

$$
\begin{aligned}
& \left|\mathscr{F}_{\theta, m}\left(\frac{n \pi}{\sigma}\right)-\widetilde{\mathscr{F}}_{\theta, m}\left(\frac{n \pi}{\sigma}\right)\right|<\varepsilon, \\
& \left|\mathscr{F}_{\theta, m}^{\prime}\left(\frac{n \pi}{\sigma}\right)-\widetilde{\mathscr{F}}_{\theta, m}^{\prime}\left(\frac{n \pi}{\sigma}\right)\right|<\varepsilon,
\end{aligned}
$$

for a sufficiently small $\varepsilon$. From $(42)$ we can see that $\mathscr{F}_{\theta, m}(\mu)$ satisfies the condition (9) when $m>4$ and therefore whenever $0<\varepsilon \leq \min \{\pi / \sigma, \sigma / \pi, 1 / \sqrt{e}\}$ we have

$$
\left|\mathscr{F}_{\theta, m, N}(\mu)-\widetilde{\mathscr{F}}_{\theta, m, N}(\mu)\right| \leq \mathscr{A}(\varepsilon), \quad \mu \in \mathbb{R},
$$

where there is a positive constant $M_{\mathscr{F}_{\theta, m}}$ for which, cf. (10),

$$
\begin{aligned}
\mathscr{A}(\varepsilon):=\frac{2 e^{1 / 4}}{\sigma}\{ & \sqrt{3} e(1+\sigma)+\left(\frac{\pi}{\sigma} A+M_{\mathscr{F}_{\theta, m}}\right) \rho(\varepsilon) \\
& \left.+(\sigma+2+\log (2)) M_{\mathscr{F}_{\theta, m}}\right\} \varepsilon \log \left(\frac{1}{\varepsilon}\right) .
\end{aligned}
$$

Here

$$
\begin{gathered}
A:=\frac{3 \sigma}{\pi}\left(\left|\mathscr{F}_{\theta, m}(0)\right|+\frac{\sigma}{\pi} M_{\mathscr{F}_{\theta, m}}\right), \\
\rho(\varepsilon):=\gamma+10 \log \left(\frac{1}{\varepsilon}\right) .
\end{gathered}
$$

In the following we use the technique of [26], where only truncation error analysis is considered to determine enclosure intervals for the eigenvalues, see also [41]. Let $\mu^{* 2}$ be an eigenvalue; that is,

$$
\Delta\left(\mu^{*}\right)=\mathscr{G}\left(\mu^{*}\right)+\left(\frac{\sin \theta \mu^{*}}{\theta \mu^{*}}\right)^{-m} \mathscr{F}_{\theta, m}\left(\mu^{*}\right)=0 .
$$

Then it follows that

$$
\begin{aligned}
\mathscr{G} & \left(\mu^{*}\right)+\left(\frac{\sin \theta \mu^{*}}{\theta \mu^{*}}\right)^{-m} \widetilde{\mathscr{F}}_{\theta, m, N}\left(\mu^{*}\right) \\
= & \left(\frac{\sin \theta \mu^{*}}{\theta \mu^{*}}\right)^{-m} \widetilde{\mathscr{F}}_{\theta, m, N}\left(\mu^{*}\right)-\left(\frac{\sin \theta \mu^{*}}{\theta \mu^{*}}\right)^{-m} \mathscr{F}_{\theta, m}\left(\mu^{*}\right) \\
= & {\left[\left(\frac{\sin \theta \mu^{*}}{\theta \mu^{*}}\right)^{-m} \widetilde{\mathscr{F}}_{\theta, m, N}\left(\mu^{*}\right)-\left(\frac{\sin \theta \mu^{*}}{\theta \mu^{*}}\right)^{-m} \mathscr{F}_{\theta, m, N}\left(\mu^{*}\right)\right] } \\
& +\left[\left(\frac{\sin \theta \mu^{*}}{\theta \mu^{*}}\right)^{-m} \mathscr{F}_{\theta, m, N}\left(\mu^{*}\right)-\left(\frac{\sin \theta \mu^{*}}{\theta \mu^{*}}\right)^{-m} \mathscr{F}_{\theta, m}\left(\mu^{*}\right)\right]
\end{aligned}
$$

and so

$$
\begin{aligned}
\mid \mathscr{G} & \left(\mu^{*}\right)+\left(\frac{\sin \theta \mu^{*}}{\theta \mu^{*}}\right)^{-m} \widetilde{\mathscr{F}}_{\theta, m, N}\left(\mu^{*}\right) \mid \\
\leq & \left|\frac{\sin \theta \mu^{*}}{\theta \mu^{*}}\right|^{-m}\left(T_{N, m-4, \sigma}\left(\mu^{*}\right)+\mathscr{A}(\varepsilon)\right) .
\end{aligned}
$$

Since $\mathscr{G}\left(\mu^{*}\right)+\left(\sin \theta \mu^{*} / \theta \mu^{*}\right)^{-m} \widetilde{\mathscr{F}}_{\theta, m, N}\left(\mu^{*}\right)$ is given and | $\sin \theta \mu^{*} /\left.\theta \mu^{*}\right|^{-m}\left(T_{N, m-4, \sigma}\left(\mu^{*}\right)+\mathscr{A}(\varepsilon)\right)$ has computable upper bound, we can define an enclosure for $\mu^{*}$ by solving the following system of inequalities:

$$
\begin{aligned}
-\left|\frac{\sin \theta \mu^{*}}{\theta \mu^{*}}\right|^{-m}\left(T_{N, m-4, \sigma}\left(\mu^{*}\right)+\mathscr{A}(\varepsilon)\right) \\
\leq \mathscr{G}\left(\mu^{*}\right)+\left(\frac{\sin \theta \mu^{*}}{\theta \mu^{*}}\right)^{-m} \widetilde{\mathscr{F}}_{\theta, m, N}\left(\mu^{*}\right) \\
\leq\left|\frac{\sin \theta \mu^{*}}{\theta \mu^{*}}\right|^{-m}\left(T_{N, m-4, \sigma}\left(\mu^{*}\right)+\mathscr{A}(\varepsilon)\right) .
\end{aligned}
$$

Its solution is an interval containing $\mu^{*}$, and over which the graph $\mathscr{G}\left(\mu^{*}\right)+\left(\sin \theta \mu^{*} / \theta \mu^{*}\right)^{-m} \widetilde{\mathscr{F}}_{\theta, m, N}\left(\mu^{*}\right)$ is squeezed between the graphs as follows:

$$
\begin{gathered}
-\left|\frac{\sin \theta \mu^{*}}{\theta \mu^{*}}\right|^{-m}\left(T_{N, m-4, \sigma}\left(\mu^{*}\right)+\mathscr{A}(\varepsilon)\right), \\
\left|\frac{\sin \theta \mu^{*}}{\theta \mu^{*}}\right|^{-m}\left(T_{N, m-4, \sigma}\left(\mu^{*}\right)+\mathscr{A}(\varepsilon)\right) .
\end{gathered}
$$

Using the fact that

$$
\widetilde{\mathscr{F}}_{\theta, m, N}(\mu) \longrightarrow \mathscr{F}_{\theta, m}(\mu)
$$


uniformly over any compact set and since $\mu^{*}$ is a simple root, we obtain the following for large $N$ and sufficiently small $\varepsilon$ :

$$
\frac{\partial}{\partial \mu}\left(\mathscr{G}(\mu)+\left(\frac{\sin \theta \mu}{\theta \mu}\right)^{-m} \widetilde{\mathscr{F}}_{\theta, m, N}(\mu)\right) \neq 0
$$

in a neighborhood of $\mu^{*}$. Hence, the graph of $\mathscr{C}(\mu)+(\sin \theta \mu / \theta \mu)^{-m} \widetilde{\mathscr{F}}_{\theta, m, N}(\mu)$ intersects the graphs $-|\sin \theta \mu / \theta \mu|^{-m}\left(T_{N, m-4, \sigma}(\mu)+\mathscr{A}(\varepsilon)\right)$ and $|\sin \theta \mu / \theta \mu|^{-m}$ $\left(T_{N, m-4, \sigma}(\mu)+\mathscr{A}(\varepsilon)\right)$ at two points with abscissae $a_{-}\left(\mu^{*}, N, \varepsilon\right) \leq a_{+}\left(\mu^{*}, N, \varepsilon\right)$ and the solution of the system of inequalities (60) is the interval

$$
I_{\varepsilon, N}:=\left[a_{-}\left(\mu^{*}, N, \varepsilon\right), a_{+}\left(\mu^{*}, N, \varepsilon\right)\right]
$$

and in particular $\mu^{*} \in I_{\varepsilon, N}$. Summarizing the above discussion, we arrive at the following lemma which is similar to that of [26].

Lemma 4. For any eigenvalue $\mu^{* 2}$, we can find $N_{0} \in \mathbb{Z}^{+}$and sufficiently small $\varepsilon$ such that $\mu^{*} \in I_{\varepsilon, N}$ for $N>N_{0}$. Moreover, we get

$$
\begin{array}{r}
{\left[a_{-}\left(\mu^{*}, N, \varepsilon\right), a_{+}\left(\mu^{*}, N, \varepsilon\right)\right] \longrightarrow\left\{\mu^{*}\right\}} \\
\text { as } N \longrightarrow \infty, \quad \varepsilon \longrightarrow 0 .
\end{array}
$$

Proof. Since all eigenvalues of $\Pi\left(q, a, b, a^{\prime}, b^{\prime}\right)$ are simple, then for large $N$ and sufficiently small $\varepsilon$ we have $(\partial / \partial \mu)\left(\mathscr{G}(\mu)+(\sin \theta \mu / \theta \mu)^{-m} \widetilde{\mathscr{F}}_{\theta, m, N}(\mu)\right)>0$, in a neighborhood of $\mu^{*}$. Choose $N_{0}$ such that

$$
\begin{aligned}
\mathscr{G}(\mu) & +\left(\frac{\sin \theta \mu}{\theta \mu}\right)^{-m} \widetilde{\mathscr{F}}_{\theta, m, N_{0}}(\mu) \\
& = \pm\left|\frac{\sin \theta \mu}{\theta \mu}\right|^{-m}\left(T_{N_{0}, m-4, \sigma}(\mu)+\mathscr{A}(\varepsilon)\right)
\end{aligned}
$$

has two distinct solutions which we denote by $a_{-}\left(\mu^{*}, N_{0}, \varepsilon\right) \leq$ $a_{+}\left(\mu^{*}, N_{0}, \varepsilon\right)$. The decay of $T_{N, m-4, \sigma}(\mu) \rightarrow 0$ as $N \rightarrow \infty$ and $\mathscr{A}(\varepsilon) \rightarrow 0$ as $\varepsilon \rightarrow 0$ will ensure the existence of the solutions $a_{-}\left(\mu^{*}, N, \varepsilon\right)$ and $a_{+}\left(\mu^{*}, N, \varepsilon\right)$ as $N \rightarrow \infty$ and $\varepsilon \rightarrow 0$. For the second point we recall that $\widetilde{\mathscr{F}}_{\theta, m, N}(\mu) \rightarrow \mathscr{F}_{\theta, m}(\mu)$ as $N \rightarrow$ $\infty$ and as $\varepsilon \rightarrow 0$. Hence, by taking the limit we obtain

$$
\begin{aligned}
& \mathscr{G}\left(a_{+}\left(\mu^{*}, \infty, 0\right)\right)+\left(\frac{\sin \theta \mu^{*}}{\theta \mu^{*}}\right)^{-m} \mathscr{F}_{\theta, m}\left(a_{+}\left(\mu^{*}, \infty, 0\right)\right)=0, \\
& \mathscr{G}\left(a_{-}\left(\mu^{*}, \infty, 0\right)\right)+\left(\frac{\sin \theta \mu^{*}}{\theta \mu^{*}}\right)^{-m} \mathscr{F}_{\theta, m}\left(a_{-}\left(\mu^{*}, \infty, 0\right)\right)=0 .
\end{aligned}
$$

That is, $\Delta\left(a_{+}\right)=\Delta\left(a_{-}\right)=0$. This leads us to conclude that $a_{+}=a_{-}=\mu^{*}$, since $\mu^{*}$ is a simple root.

Let $\widetilde{\Delta}_{N}(\mu):=\mathscr{G}(\mu)+(\sin \theta \mu / \theta \mu)^{-m} \widetilde{\mathscr{F}}_{\theta, m, N}(\mu)$. Then (50) and (54) imply

$$
\begin{array}{r}
\left|\Delta(\mu)-\widetilde{\Delta}_{N}(\mu)\right| \leq\left|\frac{\sin \theta \mu}{\theta \mu}\right|^{-m}\left(T_{N, m-4, \sigma}(\mu)+\mathscr{A}(\varepsilon)\right), \\
|\mu|<\frac{N \pi}{\sigma}
\end{array}
$$

and $\theta$ is chosen sufficiently small for which $|\theta \mu|<\pi$. Therefore, $\theta, m$ must be chosen so that for $|\mu|<N \pi / \sigma$

$$
m>4, \quad \theta \in(0,1), \quad|\theta \mu|<\pi .
$$

Let $\mu^{*}$ be an eigenvalue and let $\mu_{N}$ be its approximation. Thus, $\Delta\left(\mu^{*}\right)=0$ and $\widetilde{\Delta}_{N}\left(\mu_{N}\right)=0$. From (68) we have $\left|\widetilde{\Delta}_{N}\left(\mu^{*}\right)\right| \leq$ $\left|\sin \theta \mu^{*} / \theta \mu^{*}\right|^{-m}\left(T_{N, m-4, \sigma}\left(\mu^{*}\right)+\mathscr{A}(\varepsilon)\right)$. Now we estimate the error $\left|\mu^{*}-\mu_{N}\right|$ for an eigenvalue $\mu^{*}$.

Theorem 5. Let $\mu^{* 2}$ be an eigenvalue of $\Pi\left(q, a, b, a^{\prime}, b^{\prime}\right)$. For sufficient large $N$ we have the following estimate:

$$
\left|\mu^{*}-\mu_{N}\right|<\left|\frac{\sin \theta \mu_{N}}{\theta \mu_{N}}\right|^{-m} \frac{T_{N, m-4, \sigma}\left(\mu_{N}\right)+\mathscr{A}(\varepsilon)}{\inf _{\zeta \in I_{\varepsilon, N}}\left|\Delta^{\prime}(\zeta)\right|} .
$$

Proof. Since $\Delta\left(\mu_{N}\right)-\widetilde{\Delta}_{N}\left(\mu_{N}\right)=\Delta\left(\mu_{N}\right)-\Delta\left(\mu^{*}\right)$, then from (68) and after replacing $\mu$ by $\mu_{N}$, we obtain

$$
\left|\Delta\left(\mu_{N}\right)-\Delta\left(\mu^{*}\right)\right| \leq\left|\frac{\sin \theta \mu_{N}}{\theta \mu_{N}}\right|^{-m}\left(T_{N, m-4, \sigma}\left(\mu_{N}\right)+\mathscr{A}(\varepsilon)\right) .
$$

Using the mean value theorem yields that for some $\zeta \in J_{\mathcal{\varepsilon}, N}:=$ $\left[\min \left(\mu^{*}, \mu_{N}\right), \max \left(\mu^{*}, \mu_{N}\right)\right]$,

$$
\begin{aligned}
& \left|\left(\mu^{*}-\mu_{N}\right) \Delta^{\prime}(\zeta)\right| \\
& \quad \leq\left|\frac{\sin \theta \mu_{N}}{\theta \mu_{N}}\right|^{-m}\left(T_{N, m-4, \sigma}\left(\mu_{N}\right)+\mathscr{A}(\varepsilon)\right), \quad \zeta \in J_{\varepsilon, N} \subset I_{\varepsilon, N} .
\end{aligned}
$$

Since the eigenvalues are simple, then for sufficiently large $N \operatorname{Ninf}_{\zeta \in I_{\varepsilon, N}}\left|\Delta^{\prime}(\zeta)\right|>0$ and we get (70).

\section{The Case of $\Pi\left(q, a, b, 0, b^{\prime}\right)$}

This section includes briefly a treatment similarly to that of the previous section for the eigenvalue problem $\Pi\left(q, a, b, 0, b^{\prime}\right)$ introduced in Section 1 . Notice that condition (18) implies that the analysis of problem $\Pi\left(q, a, b, 0, b^{\prime}\right)$ is not included in that of $\Pi\left(q, a, b, a^{\prime}, b^{\prime}\right)$. Let $\psi(\cdot, \mu)$ denote the solution of (15) satisfying the following initial conditions:

$$
\psi(0, \mu)=a_{2}, \quad \psi^{\prime}(0, \mu)=-a_{1} .
$$

Thus, $\psi(\cdot, \mu)$ satisfies the boundary condition (16). The eigenvalues of the problem $\Pi\left(q, a, b, 0, b^{\prime}\right)$ are the zeros of the function as follows:

$$
\Omega(\mu):=\left(b_{1}^{\prime} \mu^{2}-b_{1}\right) \psi(1, \mu)+\left(b_{2}^{\prime} \mu^{2}-b_{2}\right) \psi^{\prime}(1, \mu) .
$$

Recall that $\Pi\left(q, a, b, a^{\prime}, b^{\prime}\right)$ has denumerable set of real and simple eigenvalues, $\mathrm{cf}$. [38]. Using the method of variation of constants, the solution $\psi(x, \mu)$ satisfies Volterra integral equation as follows:

$$
\psi(x, \mu)=a_{2} \cos \mu x-a_{1} \frac{\sin \mu x}{\mu}+T[\psi](x, \mu),
$$


where $T$ is the Volterra operator defined in (22). Differentiating (75), we get

$$
\psi^{\prime}(x, \mu)=-a_{2} \mu \sin \mu x-a_{1} \cos \mu x+\widetilde{T}[\psi](x, \mu),
$$

where $\widetilde{T}$ is the Volterra operator defined in (24). Define $h_{1}(\cdot, \mu)$ and $h_{2}(\cdot, \mu)$ to be

$$
h_{1}(x, \mu):=T[\psi](x, \mu), \quad h_{2}(x, \mu):=\widetilde{T}[\psi](x, \mu) .
$$

As in the preceding section we split $\Omega(\mu)$ into

$$
\Omega(\mu):=\mathscr{K}(\mu)+\mathscr{U}(\mu),
$$

where $\mathscr{K}(\mu)$ is the known part

$$
\begin{aligned}
\mathscr{K}(\mu)= & \left(b_{1}^{\prime} \mu^{2}-b_{1}\right)\left[a_{2} \cos \mu-a_{1} \frac{\sin \mu}{\mu}\right] \\
& +\left(b_{2}^{\prime} \mu^{2}-b_{2}\right)\left[-a_{2} \mu \sin \mu-a_{1} \cos \mu\right],
\end{aligned}
$$

and $\mathscr{U}(\mu)$ is the unknown one

$$
\mathcal{U}(\mu):=\left(b_{1}^{\prime} \mu^{2}-b_{1}\right) h_{1}(1, \mu)+\left(b_{2}^{\prime} \mu^{2}-b_{2}\right) h_{2}(1, \mu) .
$$

Then, as in the previous section, $\mathscr{U}(\mu)$ is entire in $\mu$ for each $x \in[0,1]$ for which

$$
|\mathcal{U}(\mu)| \leq \frac{c_{1} c_{3} c_{4} c_{6}\left(1+|\mu|^{2}\right)}{1+|\mu|} e^{|\Im \mu|}, \quad \mu \in \mathbb{C},
$$

where $c_{6}:=\max \left\{\left|b_{1}\right|+\left|b_{2}\right| \tau,\left|b_{1}^{\prime}\right|+\left|b_{2}^{\prime}\right| \tau\right\}$.

Let $\theta \in(0,1)$ and let $m$ be as in the previous section, but $m>2$. Define $\mathscr{R}_{m, \theta}(\mu)$ to be

$$
\mathscr{R}_{m, \theta}(\mu)=\left(\frac{\sin \theta \mu}{\theta \mu}\right)^{m} \mathscr{U}(\mu), \quad \mu \in \mathbb{C} .
$$

Hence,

$$
\left|\mathscr{R}_{m, \theta}(\mu)\right| \leq \frac{c_{0}^{m} c_{1} c_{3} c_{4} c_{6}\left(1+|\mu|^{2}\right)}{(1+\theta|\mu|)^{m+1}} e^{|\Im \mu|(1+m \theta)}, \quad \mu \in \mathbb{C},
$$

and $\mu^{m-2} \mathscr{R}_{m, \theta}(\mu) \in L^{2}(\mathbb{R})$ with

$$
\begin{aligned}
E_{m-2}\left(\mathscr{R}_{m, \theta}\right) & =\sqrt{\int_{-\infty}^{\infty}\left|\mu^{m-2} \mathscr{R}_{m, \theta}(\mu)\right|^{2} d \mu} \\
& \leq \sqrt{2} c_{0}^{m} c_{1} \mathcal{c}_{3} c_{4} c_{6} \omega_{0},
\end{aligned}
$$

where

$$
\omega_{0}:=\sqrt{\frac{12 \theta^{2} \Gamma[2 m-3]+\Gamma[2 m-1]}{\theta^{2 m-1} \Gamma[2(m+1)]}} .
$$

Thus, $\mathscr{R}_{m, \theta}(\mu)$ belongs to the Paley-Wiener space $\mathrm{PW}_{\sigma}^{2}$ with $\sigma=1+m \theta$. Since $\mathscr{R}_{\theta, m}(\mu) \in \mathrm{PW}_{\sigma}^{2} \subset \mathrm{PW}_{2 \sigma}^{2}$, then we can reconstruct the functions $\mathscr{R}_{\theta, m}(\mu)$ via the following sampling formula:

$$
\begin{aligned}
\mathscr{R}_{\theta, m}(\mu) & \\
=\sum_{n=-\infty}^{\infty}[ & \mathscr{R}_{\theta, m}\left(\frac{n \pi}{\sigma}\right) S_{n}^{2}(\mu) \\
& \left.\quad+\mathscr{R}_{\theta, m}^{\prime}\left(\frac{n \pi}{\sigma}\right) \frac{\sin (\sigma \mu-n \pi)}{\sigma} S_{n}(\mu)\right] .
\end{aligned}
$$

Let $N \in \mathbb{Z}^{+}, N>m$, and approximate $\mathscr{R}_{\theta, m}(\mu)$ by its truncated series $\mathscr{R}_{\theta, m, N}(\mu)$, where

$$
\begin{aligned}
\mathscr{R}_{\theta, m, N}(\mu) & \\
:=\sum_{n=-N}^{N}[ & \mathscr{R}_{\theta, m}\left(\frac{n \pi}{\sigma}\right) S_{n}^{2}(\mu) \\
& \left.\quad+\mathscr{R}_{\theta, m}^{\prime}\left(\frac{n \pi}{\sigma}\right) \frac{\sin (\sigma \mu-n \pi)}{\sigma} S_{n}(\mu)\right] .
\end{aligned}
$$

Since all eigenvalues are real, then from now on we restrict ourselves to $\mu \in \mathbb{R}$. Since $\mu^{m-2} \mathscr{R}_{\theta, m}(\mu) \in L^{2}(\mathbb{R})$, the truncation error, cf. (5), is given for $|\mu|<N \pi / \sigma$ by

$$
\left|\mathscr{R}_{\theta, m}(\mu)-\mathscr{R}_{\theta, m, N}(\mu)\right| \leq T_{N, m-2, \sigma}(\mu),
$$

where

$$
\begin{aligned}
T_{N, m-2, \sigma}(\mu) & \\
:= & \frac{\xi_{m-2, \sigma} E_{m-2}|\sin \sigma \mu|^{2}}{\sqrt{3}(N+1)^{m-2}}\left(\frac{1}{(N \pi-\sigma \mu)^{3 / 2}}+\frac{1}{(N \pi+\sigma \mu)^{3 / 2}}\right) \\
& +\frac{\xi_{m-2, \sigma}\left(\sigma E_{m-2}+(m-2) E_{m-3}\right)|\sin \sigma \mu|^{2}}{\sigma(N+1)^{m-2}} \\
& \times\left(\frac{1}{\sqrt{N \pi-\sigma \mu}}+\frac{1}{\sqrt{N \pi+\sigma \mu}}\right) .
\end{aligned}
$$

The samples $\left\{\mathscr{R}_{\theta, m}(n \pi / \sigma)\right\}_{n=-N}^{N}$ and $\left\{\mathscr{R}_{\theta, m}^{\prime}(n \pi / \sigma)\right\}_{n=-N}^{N}$, in general, are not known explicitly. So we approximate them by solving numerically $4 N+2$ initial value problems at the nodes $\{n \pi / \sigma\}_{n=-N}^{N}$. Let $\left\{\widetilde{\mathscr{R}}_{\theta, m}(n \pi / \sigma)\right\}_{n=-N}^{N}$ and let $\left\{\widetilde{\mathscr{R}}_{\theta, m}^{\prime}(n \pi / \sigma)\right\}_{n=-N}^{N}$ be the approximations of the samples of $\left\{\mathscr{R}_{\theta, m}(n \pi / \sigma)\right\}_{n=-N}^{N}$ and $\left\{\mathscr{R}_{\theta, m}^{\prime}(n \pi / \sigma)\right\}_{n=-N}^{N}$, respectively. Now we define $\widetilde{\mathscr{R}}_{\theta, m, N}(\mu)$, which approximates $\mathscr{R}_{\theta, m, N}(\mu)$

$$
\widetilde{\mathscr{R}}_{\theta, m, N}(\mu)
$$

$$
\begin{aligned}
:=\sum_{n=-N}^{N}[ & \widetilde{\mathscr{R}}_{\theta, m}\left(\frac{n \pi}{\sigma}\right) S_{n}^{2}(\mu) \\
& \left.+\widetilde{\mathscr{R}}_{\theta, m}^{\prime}\left(\frac{n \pi}{\sigma}\right) \frac{\sin (\sigma \mu-n \pi)}{\sigma} S_{n}(\mu)\right],
\end{aligned}
$$


Using standard methods for solving initial problems, we may assume that for $|n|<N$

$$
\begin{aligned}
& \left|\mathscr{R}_{\theta, m}\left(\frac{n \pi}{\sigma}\right)-\widetilde{\mathscr{R}}_{\theta, m}\left(\frac{n \pi}{\sigma}\right)\right|<\varepsilon, \\
& \left|\mathscr{R}_{\theta, m}^{\prime}\left(\frac{n \pi}{\sigma}\right)-\widetilde{\mathscr{R}}_{\theta, m}^{\prime}\left(\frac{n \pi}{\sigma}\right)\right|<\varepsilon,
\end{aligned}
$$

for a sufficiently small $\varepsilon$. From $(83)$ we can see that $\mathscr{R}_{\theta, m}(\mu)$ satisfies the condition (9) when $m>2$ and therefore whenever $0<\varepsilon \leq \min \{\pi / \sigma, \sigma / \pi, 1 / \sqrt{e}\}$ we have

$$
\left|\mathscr{R}_{\theta, m, N}(\mu)-\widetilde{\mathscr{R}}_{\theta, m, N}(\mu)\right| \leq \mathscr{A}(\varepsilon), \quad \mu \in \mathbb{R},
$$

where there is a positive constant $M_{\mathscr{R}_{\theta, m}}$ for which, cf. (10), and

$$
\begin{aligned}
\mathscr{A}(\varepsilon):=\frac{2 e^{1 / 4}}{\sigma}\{ & \sqrt{3} e(1+\sigma)+\left(\frac{\pi}{\sigma} A+M_{\mathscr{R}_{\theta, m}}\right) \rho(\varepsilon) \\
& \left.+(\sigma+2+\log (2)) M_{\mathscr{R}_{\theta, m}}\right\} \varepsilon \log \left(\frac{1}{\varepsilon}\right) .
\end{aligned}
$$

Here

$$
\begin{gathered}
A:=\frac{3 \sigma}{\pi}\left(\left|\mathscr{R}_{\theta, m}(0)\right|+\frac{\sigma}{\pi} M_{\mathscr{R}_{\theta, m}}\right), \\
\rho(\varepsilon):=\gamma+10 \log \left(\frac{1}{\varepsilon}\right) .
\end{gathered}
$$

As in the above section, we have the following lemma.

Lemma 6. For any eigenvalue $\mu^{* 2}$ of the problem $\Pi\left(q, a, b, 0, b^{\prime}\right)$, we can find $N_{0} \in \mathbb{Z}^{+}$and sufficiently small $\varepsilon$ such that $\mu^{*} \in \mathscr{I}_{\varepsilon, N}$ for $N>N_{0}$, where

$$
\mathscr{I}_{\varepsilon, N}:=\left[b_{-}\left(\mu^{*}, N, \varepsilon\right), a b_{+}\left(\mu^{*}, N, \varepsilon\right)\right]
$$

$b_{-}, b_{+}$are the solutions of the inequalities

$$
\begin{aligned}
& -\left|\frac{\sin \theta \mu}{\theta \mu}\right|^{-m}\left(T_{N, m-2, \sigma}(\mu)+\mathscr{A}(\varepsilon)\right) \\
& \quad \leq \widetilde{\Omega}_{N}(\mu) \leq\left|\frac{\sin \theta \mu}{\theta \mu}\right|^{-m}\left(T_{N, m-2, \sigma}(\mu)+\mathscr{A}(\varepsilon)\right) .
\end{aligned}
$$

Moreover, we get

$$
\begin{array}{r}
{\left[b_{-}\left(\mu^{*}, N, \varepsilon\right), b_{+}\left(\mu^{*}, N, \varepsilon\right)\right] \longrightarrow\left\{\mu^{*}\right\}} \\
\text { as } N \longrightarrow \infty, \quad \varepsilon \longrightarrow 0
\end{array}
$$

Let $\widetilde{\Omega}_{N}(\mu):=\mathscr{K}(\mu)+(\sin \theta \mu / \theta \mu)^{-m} \widetilde{\mathscr{R}}_{\theta, m, N}(\mu)$. Then $(88)$ and (92) imply

$$
\begin{aligned}
& \left|\Omega(\mu)-\widetilde{\Omega}_{N}(\mu)\right| \\
& \quad \leq\left|\frac{\sin \theta \mu}{\theta \mu}\right|^{-m}\left(T_{N, m-2, \sigma}(\mu)+\mathscr{A}(\varepsilon)\right), \quad|\mu|<\frac{N \pi}{\sigma}
\end{aligned}
$$

and $\theta$ is chosen sufficiently small for which $|\theta \mu|<\pi$. Therefore, $\theta, m$ must be chosen so that for $|\mu|<N \pi / \sigma$

$$
m>2, \quad \theta \in(0,1), \quad|\theta \mu|<\pi .
$$

Let $\mu^{*}$ be an eigenvalue and $\mu_{N}$ be its approximation. Thus $\Omega\left(\mu^{*}\right)=0$ and $\widetilde{\Omega}_{N}\left(\mu_{N}\right)=0$. From (98) we have $\left|\widetilde{\Omega}_{N}\left(\mu^{*}\right)\right| \leq$ $\left|\sin \theta \mu^{*} / \theta \mu^{*}\right|^{-m}\left(T_{N, m-2, \sigma}\left(\mu^{*}\right)+\mathscr{A}(\varepsilon)\right)$. Now we estimate the error $\left|\mu^{*}-\mu_{N}\right|$ for an eigenvalue $\mu^{*}$. Finally we have the following estimate.

Theorem 7. Let $\mu^{* 2}$ be an eigenvalue of the problem $\Pi\left(q, a, b, 0, b^{\prime}\right)$. For sufficient large $N$ we have the following estimate

$$
\left|\mu^{*}-\mu_{N}\right|<\left|\frac{\sin \theta \mu_{N}}{\theta \mu_{N}}\right|^{-m} \frac{T_{N, m-2, \sigma}\left(\mu_{N}\right)+\mathscr{A}(\varepsilon)}{\inf _{\zeta \in \mathcal{F}_{\varepsilon, N}}\left|\Omega^{\prime}(\zeta)\right|} .
$$

\section{Numerical Examples}

This section includes two detailed worked examples illustrating the above technique. Examples 1 and 2 computed in $[27,45]$ with the classical sinc method, where only truncation error analysis is considered, respectively. It is clearly seen that our new method (Hermite interpolations) gives remarkably better results than in $[27,45]$, see also [41-43]. We indicate in these examples the effect of the amplitude error in the method by determining enclosure intervals for different values of $\varepsilon$. We also indicate the effect of the parameters $m$ and $\theta$ by several choices. Each example is exhibited via figures that accurately illustrate the procedure near to some of the approximated eigenvalues. More explanations are given below. Recall that $a_{ \pm}(\mu)$ and $b_{ \pm}(\mu)$ are defined by

$$
\begin{array}{r}
a_{ \pm}(\mu)=\widetilde{\Delta}_{N}(\mu) \pm\left|\frac{\sin \theta \mu}{\theta \mu}\right|^{-m}\left(T_{N, m-3, \sigma}(\mu)+\mathscr{A}(\varepsilon)\right), \\
|\mu|<\frac{N \pi}{\sigma}, \\
b_{ \pm}(\mu)=\widetilde{\Omega}_{N}(\mu) \pm\left|\frac{\sin \theta \mu}{\theta \mu}\right|^{-m}\left(T_{N, m-2, \sigma}(\mu)+\mathscr{A}(\varepsilon)\right), \\
|\mu|<\frac{N \pi}{\sigma},
\end{array}
$$

respectively. Recall also that the enclosure intervals $I_{\varepsilon, N}:=$ $\left[a_{-}, a_{+}\right]$and $\mathscr{I}_{\varepsilon, N}:=\left[b_{-}, b_{+}\right]$are determined by solving

$$
\begin{aligned}
& a_{ \pm}(\mu)=0, \quad|\mu|<\frac{N \pi}{\sigma}, \\
& b_{ \pm}(\mu)=0, \quad|\mu|<\frac{N \pi}{\sigma} .
\end{aligned}
$$

respectively. We would like to mention that Mathematica has been used to obtain the exact values for the three examples where eigenvalues cannot be computed concretely. Mathematica is also used in rounding the exact eigenvalues, which are square roots. 


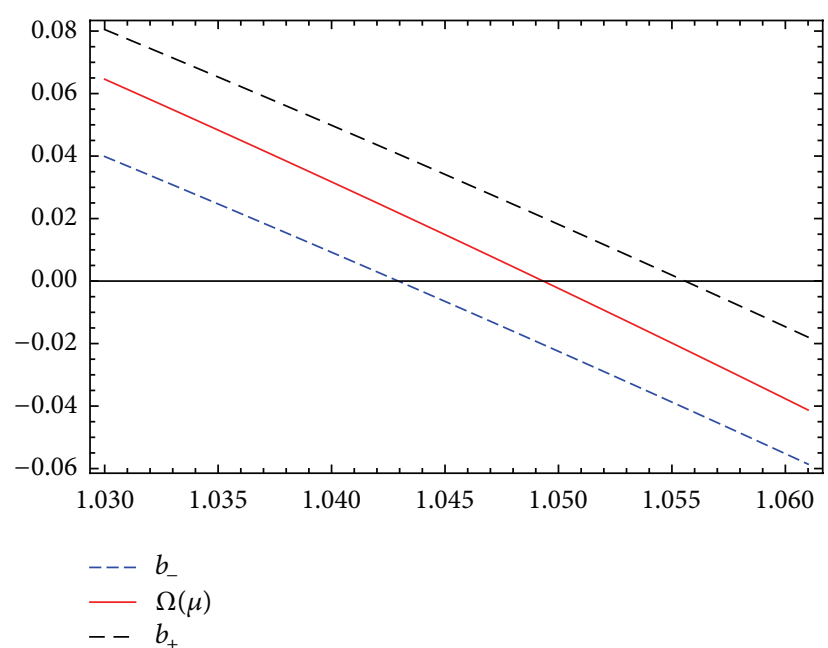

Figure 1: The enclosure interval dominating $\mu_{1}$ for $m=10, N=15$, $\theta=1 / 5$, and $\varepsilon=10^{-5}$.

Example 1. The boundary value problem [27]

$$
\begin{gathered}
-y^{\prime \prime}(x, \mu)-y(x, \mu)=\mu^{2} y(x, \mu), \quad 0 \leq x \leq 1, \\
y^{\prime}(0, \mu)=0, \\
y(1, \mu)+y^{\prime}(1, \mu)=\mu^{2}\left(2 y(1, \mu)+y^{\prime}(1, \mu)\right),
\end{gathered}
$$

is a special case of the problem treated in the previous section with $a_{1}=0, a_{2}=b_{1}=b_{2}=b_{2}^{\prime}=1, b_{1}^{\prime}=2$ and $q(x)=-1$. The characteristic function is

$$
\begin{aligned}
\Omega(\mu)= & \left(-1+2 \mu^{2}\right) \cos \left[\sqrt{1+\mu^{2}}\right] \\
& -\left(-1+\mu^{2}\right) \sqrt{1+\mu^{2}} \sin \left[\sqrt{1+\mu^{2}}\right] .
\end{aligned}
$$

The function $\mathscr{K}(\mu)$ will be

$$
\mathscr{K}(\mu)=\left(-1+2 \mu^{2}\right) \cos [\mu]-\mu\left(-1+\mu^{2}\right) \sin [\mu] .
$$

As is clearly seen, the eigenvalues cannot be computed explicitly. Tables 1, 2, and 3 indicate the application of our technique to this problem and the effect of $m, \theta$ and $\varepsilon$. By exact we mean the zeros of $\Omega(\mu)$ computed by Mathematica.

Figures 1 and 2 illustrate the enclosure intervals dominating $\mu_{1}$ for $N=15, m=10, \theta=1 / 5$, and $\varepsilon=10^{-5}$ and $\varepsilon=10^{-10}$ respectively. The middle curve represents $\Omega(\mu)$, while the upper and lower curves represent the curves of $b_{+}(\mu), b_{-}(\mu)$, respectively. We notice that when $\varepsilon=10^{-10}$, the two curves are almost identical. Similarly, Figures 3 and 4 illustrate the enclosure intervals dominating $\mu_{2}$ for $N=15, m=10, \theta=1 / 5$, and $\varepsilon=10^{-5}$ and $\varepsilon=10^{-10}$ respectively.

Example 2. The boundary value problem [45]

$$
\begin{gathered}
-y^{\prime \prime}(x, \mu)-y(x, \mu)=\mu^{2} y(x, \mu) \quad 0 \leq x \leq 1, \\
y(0, \mu)=\mu^{2} y^{\prime}(0, \mu), \quad y^{\prime}(1, \mu)=\mu^{2} y(1, \mu),
\end{gathered}
$$

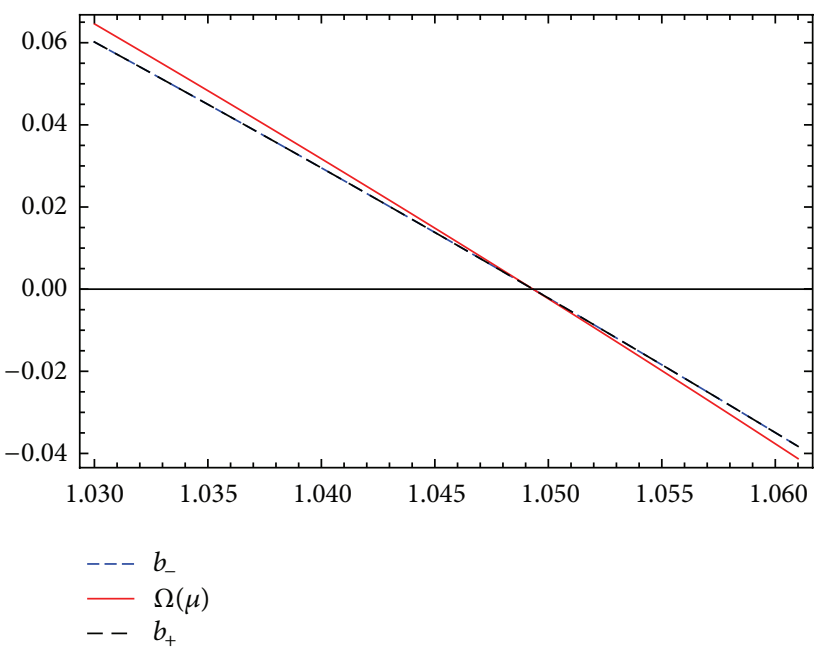

FIgURE 2: The enclosure interval dominating $\mu_{1}$ for $m=10, N=15$, $\theta=1 / 5$, and $\varepsilon=10^{-10}$.

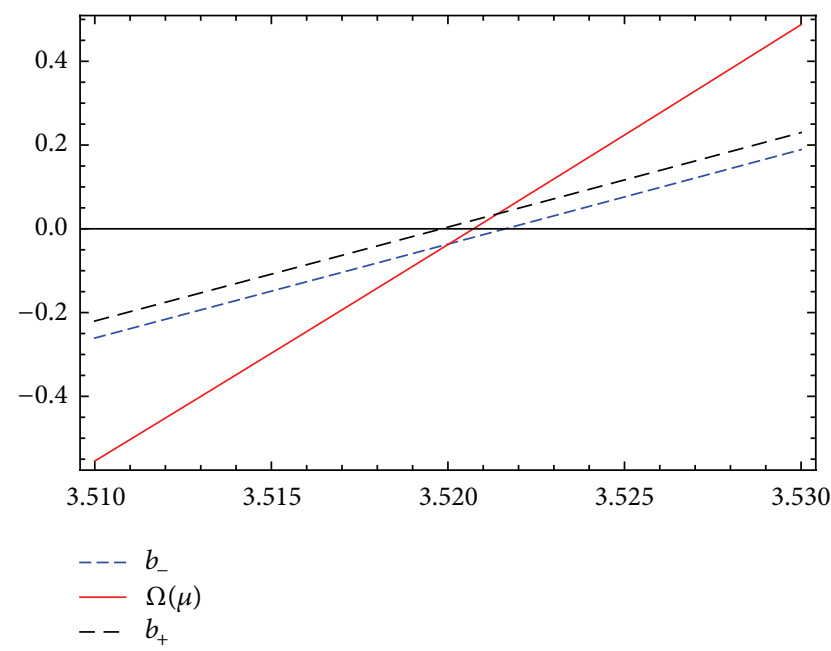

FIgURE 3: The enclosure interval dominating $\mu_{2}$ for $m=10, N=15$, $\theta=1 / 5$, and $\varepsilon=10^{-5}$.

is a special case of problem $\Pi\left(q, a, b, a^{\prime}, b^{\prime}\right)$, when $q(x)=-1$, $a_{2}=a_{1}^{\prime}=b_{1}=b_{2}^{\prime}=0$, and $a_{1}=a_{2}^{\prime}=b_{1}^{\prime}=b_{2}=1$. Here the characteristic function is

$$
\Delta(\mu)=\left(1-\mu^{4}\right) \cos \sqrt{\mu^{2}+1}-\left(2 \mu^{2}+\mu^{4}\right) \frac{\sin \sqrt{\mu^{2}+1}}{\sqrt{\mu^{2}+1}} .
$$

After computing $\mathscr{G}(\mu)$, we obtain

$$
\mathscr{G}(\mu)=\left(1+\mu^{2}\right)\left(\left(1-\mu^{2}\right) \cos \mu-\mu \sin \mu\right) .
$$

As is clearly seen, the eigenvalues cannot be computed explicitly. As in the previous example, Figures 5, 6, 7, and 8 illustrate the results of Tables 4, 5, 6, and 7 . 
TABLE 1: With $N=15$, the approximation $\mu_{k, N}$ and the exact solution $\mu_{k}$ for different choices of $m$ and $\theta$.

\begin{tabular}{lcccc}
\hline$\mu_{k}$ & $\mu_{1}$ & $\mu_{2}$ & $\mu_{3}$ & $\mu_{4}$ \\
\hline Exact $\mu_{k}$ & 1.0493258679653497 & 3.5207214555369464 & 6.505146961583527 & 9.578576417519093 \\
$\mu_{k, N}$ & & & & \\
$\quad m=6$ & & & & \\
$\quad \theta=1 / 9$ & 1.0493258487568435 & 3.5207214557864277 & 6.5051469611825405 & 9.578576417536349 \\
$\theta=1 / 12$ & 1.0493291624256957 & 3.5207212860735546 & 6.5051469673141336 & 9.578576393056661 \\
$m=10$ & & & & \\
$\theta=1 / 5$ & 1.0493258679653554 & 3.5207214555369624 & 6.505146961583516 & 9.57857641751921 \\
$\theta=1 / 8$ & 1.049325865990155 & 3.520721455899672 & 6.505146961464727 & 9.578576417559768 \\
\hline
\end{tabular}

TABLE 2: Absolute error $\left|\mu_{k}-\mu_{k, N}\right|$.

\begin{tabular}{ccccc}
\hline$\mu_{k}$ & $\mu_{1}$ & $\mu_{2}$ & $\mu_{3}$ & $\mu_{4}$ \\
\hline$m=6$ & & & & \\
$\theta=1 / 9$ & $1.92085 \times 10^{-8}$ & $2.49481 \times 10^{-10}$ & $4.00987 \times 10^{-10}$ & \\
$\theta=1 / 12$ & $3.29446 \times 10^{-6}$ & $1.69463 \times 10^{-7}$ & $5.73061 \times 10^{-9}$ & \\
$m=10$ & & & & \\
$\theta=1 / 5$ & $5.77316 \times 10^{-15}$ & $1.59872 \times 10^{-14}$ & $1.15463 \times 10^{-14}$ & \\
$\theta=1 / 8$ & $1.97519 \times 10^{-9}$ & $3.62725 \times 10^{-10}$ & $1.188 \times 10^{-10}$ & $4.1724 \times 10^{-13}$ \\
\hline
\end{tabular}

TABle 3: For $N=15, m=10$, and $\theta=1 / 5$, the exact solution $\mu_{k}$ are all inside the interval $\left[b_{-}, b_{+}\right]$for different values of $\varepsilon$.

\begin{tabular}{lcccc}
\hline$\mu_{k}$ & $\mu_{1}$ & $\mu_{2}$ & $\mu_{3}$ & $\mu_{4}$ \\
\hline Exact $\mu_{k}$ & 1.0493258679653497 & 3.5207214555369464 & 6.505146961583527 & 9.578576417519093 \\
$\mathscr{I}_{\varepsilon, N}, \varepsilon=10^{-5}$ & {$[1.04294069,1.05557896]$} & {$[3.51981844,3.52162396]$} & {$[6.50375768,6.50653831]$} & {$[9.55222305,9.60712093]$} \\
$\mathscr{I}_{\varepsilon, N}, \varepsilon=10^{-10}$ & {$[1.04932561,1.049326118]$} & {$[3.52072141,3.52072149]$} & {$[6.50514690,6.50514702]$} & {$[9.57857529,9.57857754]$} \\
\hline
\end{tabular}

$E_{8}\left(\mathscr{R}_{\theta, m}\right)=4.51845 \times 10^{8}, E_{7}\left(\mathscr{R}_{\theta, m}\right)=2.29709 \times 10^{5}, v=1, M_{\mathscr{R}_{\theta, m}}=4.55609 \times 10^{4}$.

TABLE 4: With $N=40$, the approximation $\mu_{k, N}$ and the exact solution $\mu_{k}$ for different choices of $\theta$.

\begin{tabular}{lcccc}
\hline$\mu_{k}$ & $\mu_{1}$ & $\mu_{2}$ & $\mu_{3}$ & $\mu_{4}$ \\
\hline Exact $\mu_{k}$ & 0.4828692021748484 & 1.966318052350425 & 4.827089429919572 & 7.919684444168381 \\
$\mu_{k, N}$ & & & & \\
$\quad m=8$ & & & & \\
$\quad \theta=1 / 32$ & 0.48286920221045176 & 1.96631805234574 & 4.827089429919605 & 7.919684444168366 \\
$\quad \theta=1 / 35$ & 0.4828692337692527 & 1.966318047624416 & 4.8270894299720776 & 7.91968444416245 \\
\hline
\end{tabular}

TABLE 5: Absolute error $\left|\mu_{k}-\mu_{k, N}\right|$.

\begin{tabular}{lcccc}
\hline$\mu_{k}$ & $\mu_{1}$ & $\mu_{2}$ & $\mu_{3}$ & $\mu_{4}$ \\
\hline$m=8$ & & & & \\
$\theta=1 / 32$ & $3.56034 \times 10^{-11}$ & $4.68492 \times 10^{-12}$ & $3.28626 \times 10^{-14}$ & \\
$\theta=1 / 35$ & $3.15944 \times 10^{-8}$ & $4.72601 \times 10^{-9}$ & $5.25047 \times 10^{-11}$ & $5.93126 \times 10^{-12}$ \\
\hline
\end{tabular}

TABLE 6: The approximation $\mu_{k, N}$ and the exact solution $\mu_{k}$ for $N=40, m=14$ and $\theta=1 / 26$.

\begin{tabular}{cccc}
\hline$\mu_{k}$ & exact $\mu_{k}$ & $\mu_{k, N}$ & $\left|\mu_{k}-\mu_{k, N}\right|$ \\
\hline$\mu_{1}$ & 0.4828692021748484698568637 & 0.4828692021748484678442680 & $2.012596 \times 10^{-18}$ \\
$\mu_{2}$ & 1.966318052350424642326091 & 1.9663180523504246423320204 & $5.93 \times 10^{-21}$ \\
$\mu_{3}$ & 4.8270894299195722717631337 & 4.8270894299195722717463715 & $1.6762 \times 10^{-20}$ \\
$\mu_{4}$ & 7.91968444441683813942255769 & 7.9196844441683813942260057 & $4.29 \times 10^{-22}$ \\
\hline
\end{tabular}

TABLE 7: For $N=40, m=14$ and $\theta=1 / 26$, the exact solution $\mu_{k}$ are all inside the interval $\left[a_{-}, a_{+}\right]$for different values of $\varepsilon$.

\begin{tabular}{lcccc}
\hline$\mu_{k}$ & $\mu_{1}$ & $\mu_{2}$ & $\mu_{3}$ & $\mu_{4}$ \\
\hline Exact $\mu_{k}$ & 0.4828692021748484 & 1.966318052350425 & 4.827089429919572 & 7.919684444168381 \\
$\mathscr{I}_{\varepsilon, N}, \varepsilon=10^{-5}$ & {$[0.47918888,0.48651557]$} & {$[1.96592879,1.96670680]$} & {$[4.82707252,4.82710633]$} & {$[7.91968171,7.919687175]$} \\
$\mathscr{I}_{\varepsilon, N}, \varepsilon=10^{-10}$ & {$[0.48284084,0.48289756]$} & {$[1.96631794,1.96631815]$} & {$[4.82708919,4.82708966]$} & {$[7.919684437,7.919684450]$} \\
\hline
\end{tabular}

$E_{10}\left(\mathscr{F}_{\theta, m}\right)=2.83057 \times 10^{18}, E_{9}\left(\mathscr{F}_{\theta, m}\right)=1.12829 \times 10^{14}, v=1, M_{\mathscr{F}_{\theta, m}}=1.57716 \times 10^{7}$. 


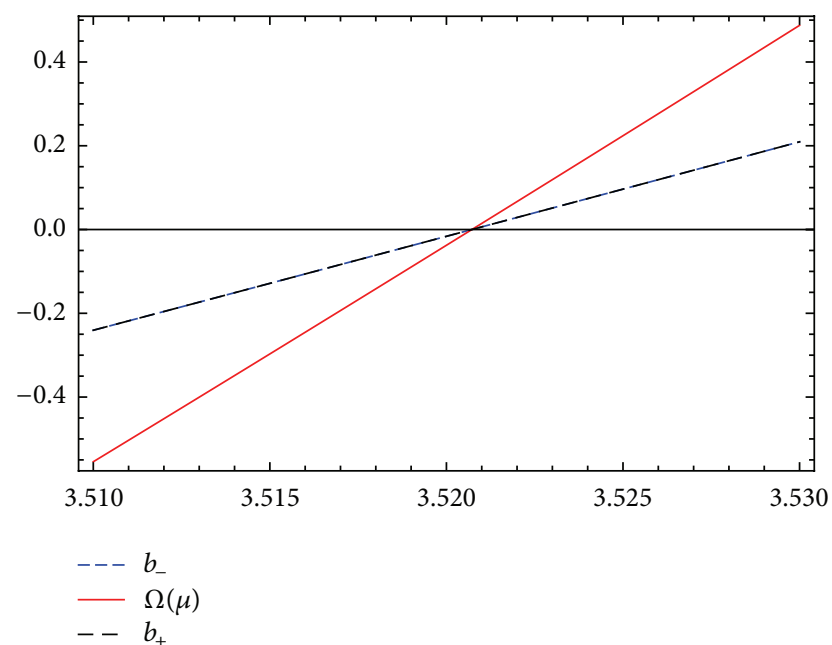

FIGURE 4: The enclosure interval dominating $\mu_{2}$ for $m=10, N=15$, $\theta=1 / 5$, and $\varepsilon=10^{-10}$.

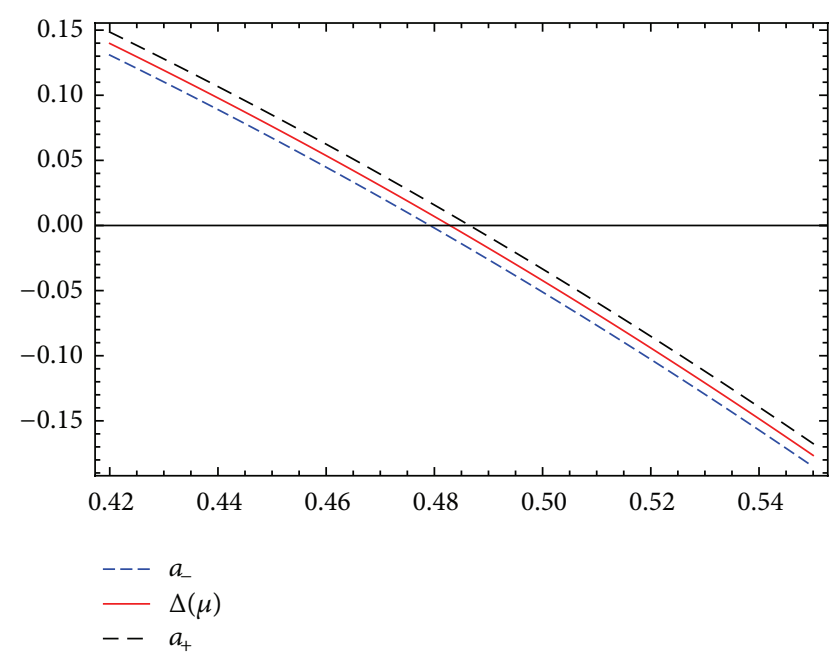

FIGURE 5: The enclosure interval dominating $\mu_{1}$ for $m=14, N=40$, $\theta=1 / 26$, and $\varepsilon=10^{-5}$.

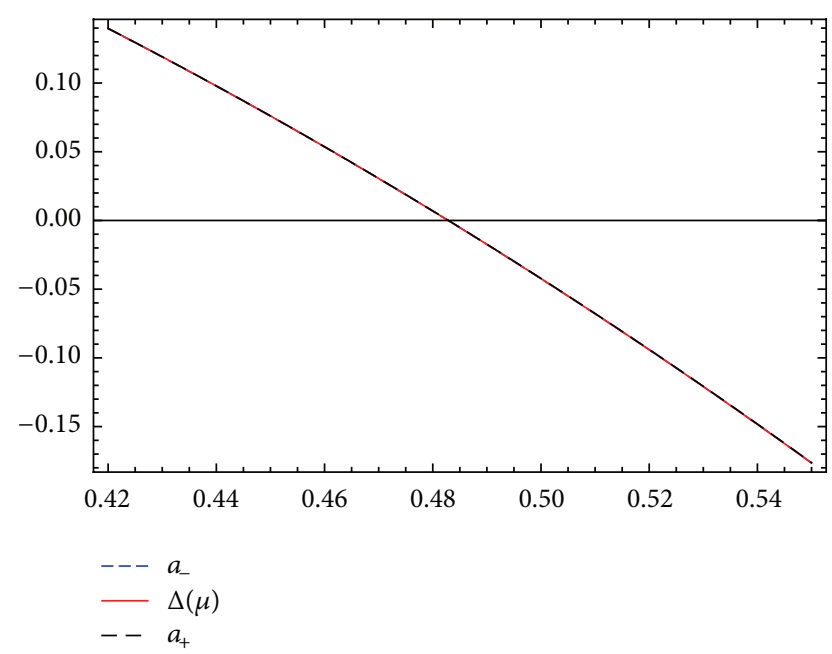

FIgURE 6: The enclosure interval dominating $\mu_{1}$ for $m=14, N=40$, $\theta=1 / 26$, and $\varepsilon=10^{-10}$.

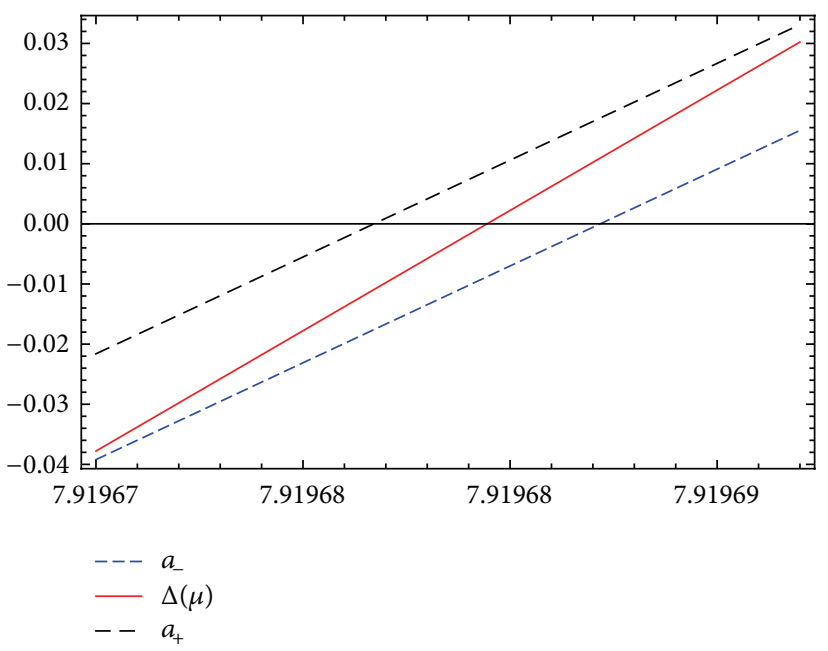

FIGURE 7: The enclosure interval dominating $\mu_{4}$ for $m=14, N=40$, $\theta=1 / 26$, and $\varepsilon=10^{-5}$.

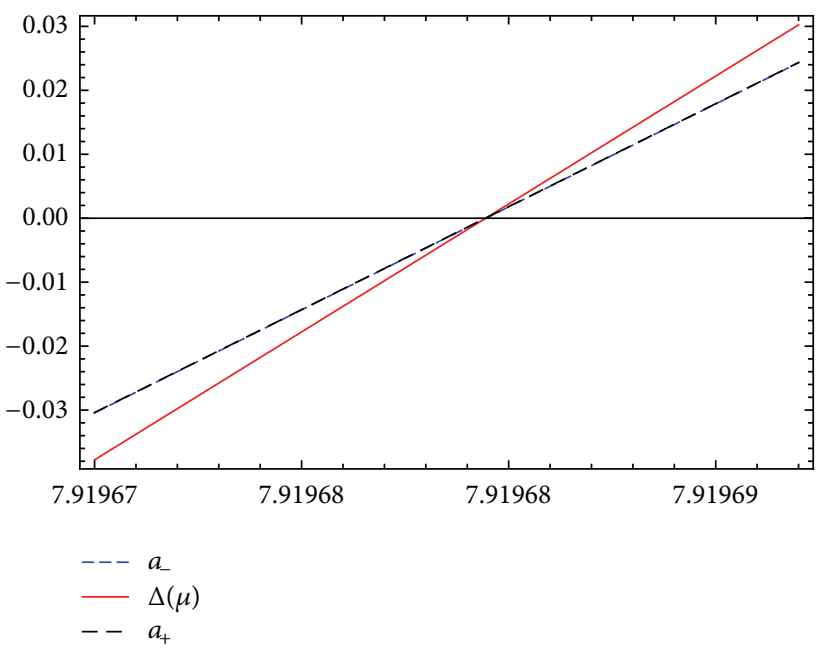

FIgURE 8: The enclosure interval dominating $\mu_{4}$ for $m=14, N=40$, $\theta=1 / 26$, and $\varepsilon=10^{-10}$.

\section{Acknowledgment}

This research has been supported by a grant from the Institute of Scientific Research at Umm AL-Qura University, Saudi Arabia.

\section{References}

[1] E. H. Doha, A. H. Bhrawy, and R. M. Hafez, "A JacobiJacobi dual-Petrov-Galerkin method for third- and fifth-order differential equations," Mathematical and Computer Modelling, vol. 53, no. 9-10, pp. 1820-1832, 2011.

[2] E. H. Doha, A. H. Bhrawy, and M. A. Saker, "Integrals of Bernstein polynomials: an application for the solution of high even-order differential equations," Applied Mathematics Letters, vol. 24, no. 4, pp. 559-565, 2011.

[3] E. Tohidi, A. H. Bhrawy, and K. Erfani, "A collocation method based on Bernoulli operational matrix for numerical solution of generalized pantograph equation," Applied Mathematical 
Modelling, vol. 37, no. 6, pp. 4283-4294, 2013.

[4] E. H. Doha and A. H. Bhrawy, "Efficient spectral-Galerkin algorithms for direct solution for second-order differential equations using Jacobi polynomials," Numerical Algorithms, vol. 42, no. 2, pp. 137-164, 2006.

[5] A. H. Bhrawy, "A Jacobi-Gauss-Lobatto collocation method for solving generalized Fitzhugh-Nagumo equation with timedependent coefficients," Applied Mathematics and Computation, vol. 222, pp. 255-264, 2013.

[6] A. H. Bhrawy, M. A. Abdelkawy, and A. Biswas, "Cnoidal and snoidal wave solutions to coupled nonlinear wave equations by the extended Jacobi's elliptic function method," Communications in Nonlinear Science and Numerical Simulation, vol. 18, no. 4, pp. 915-925, 2013.

[7] A. Imani, A. Aminataei, and A. Imani, "Collocation method via Jacobi polynomials for solving nonlinear ordinary differential equations," International Journal of Mathematics and Mathematical Sciences, vol. 2011, Article ID 673085, 11 pages, 2011.

[8] D. Baleanu, K. Diethelm, E. Scalas, and J. J. Trujillo, Fractional Calculus: Models and Numerical Methods, vol. 3 of Series on Complexity, Nonlinearity and Chaos, World Scientific, Hackensack, NJ, USA, 2012.

[9] D. Baleanu, A. H. Bhrawy, and T. M. Taha, "Two efficient generalized Laguerre spectral algorithms for fractional initial value problems," Abstract and Applied Analysis, vol. 2013, Article ID 546502, 10 pages, 2013.

[10] D. Baleanu, A. H. Bhrawy, and T. M. Taha, "A modified generalized Laguerre spectral methods for fractional differential equations on the half line," Abstract and Applied Analysis, vol. 2013, Article ID 413529, 12 pages, 2013.

[11] F. Ghaemi, R. Yunus, A. Ahmadian, S. Salahshour, M. B. Suleiman, and S. F. Saleh, "Application of fuzzy fractional kinetic equations to modelling of the acid hydrolysis reaction," Abstract and Applied Analysis, vol. 2013, Article ID 610314, 19 pages, 2013.

[12] A. H. Bhrawy and A. S. Alofi, "The operational matrix of fractional integration for shifted Chebyshev polynomials," Applied Mathematics Letters, vol. 26, no. 1, pp. 25-31, 2013.

[13] A. H. Bhrawy and M. M. Al-Shomrani, "A shifted Legendre spectral method for fractional-order multi-point boundary value problems," Advances in Difference Equations, vol. 2012, p. 8, 2012.

[14] M. H. Annaby and R. M. Asharabi, "Computing eigenvalues of Sturm-Liouville problems by Hermite interpolations," Numerical Algorithms, vol. 60, no. 3, pp. 355-367, 2012.

[15] M. M. Tharwat, A. H. Bhrawy, and A. S. Alofi, "Computing eigenvalues of discontinuous Sturm-Liouville problems with eigenparameter in all boundary conditions using Hermite approximation," Abstract and Applied Analysis, vol. 2013, Article ID 498457, 14 pages, 2013.

[16] M. M. Tharwat, A. H. Bhrawy, and A. S. Alofi, "Approximation of eigenvalues of discontinuous Sturm-Liouville problems with eigenparameter in all boundary conditions," Boundary Value Problems, vol. 2013, p. 132, 2013.

[17] M. M. Tharwat, A. H. Bhrawy, and A. Yildirim, "Numerical computation of eigenvalues of discontinuous Sturm-Liouville problems with parameter dependent boundary conditions using Sinc method," Numerical Algorithms, vol. 63, no. 1, pp. 2748, 2013.

[18] G. R. Grozev and Q. I. Rahman, "Reconstruction of entire functions from irregularly spaced sample points," Canadian Journal of Mathematics, vol. 48, no. 4, pp. 777-793, 1996.
[19] J. R. Higgins, G. Schmeisser, and J. J. Voss, "The sampling theorem and several equivalent results in analysis," Journal of Computational Analysis and Applications, vol. 2, no. 4, pp. 333371, 2000.

[20] G. Hinsen, "Irregular sampling of bandlimited $L^{p}$-functions," Journal of Approximation Theory, vol. 72, no. 3, pp. 346-364, 1993.

[21] D. Jagerman and L. Fogel, "Some general aspects of the sampling theorem," IRE Transactions on Information Theory, vol. 2, pp. 139-146, 1956.

[22] M. H. Annaby and R. M. Asharabi, "Error analysis associated with uniform Hermite interpolations of bandlimited functions," Journal of the Korean Mathematical Society, vol. 47, no. 6, pp. 1299-1316, 2010.

[23] J. R. Higgins, Sampling Theory in Fourier and Signal Analysis: Foundations, Oxford University Press, Oxford, UK, 1996.

[24] P. L. Butzer, J. R. Higgins, and R. L. Stens, "Sampling theory of signal analysis," in Development of Mathematics 1950-2000, pp. 193-234, Birkhäuser, Basel, Switzerland, 2000.

[25] P. L. Butzer, G. Schmeisser, and R. L. Stens, "An introduction to sampling analysis," in Non Uniform Sampling: Theory and Practices, F. Marvasti, Ed., pp. 17-121, Kluwer, New York, NY, USA, 2001.

[26] A. Boumenir, "Higher approximation of eigenvalues by the sampling method," BIT Numerical Mathematics, vol. 40, no. 2, pp. 215-225, 2000.

[27] A. Boumenir, "The sampling method for Sturm-Liouville problems with the eigenvalue parameter in the boundary condition," Numerical Functional Analysis and Optimization, vol. 21, no. 1-2, pp. 67-75, 2000.

[28] A. Boumenir and B. Chanane, "Eigenvalues of Sturm-Liouville systems using sampling theory," Applied Analysis, vol. 62, pp. 323-334, 1996.

[29] M. M. Tharwat, A. H. Bhrawy, and A. Yildirim, "Numerical computation of the eigenvalues of a discontinuous Dirac system using the Sinc method with error analysis," International Journal of Computer Mathematics, vol. 89, no. 15, pp. 2061-2080, 2012.

[30] J. Lund and K. Bowers, Sinc Methods for Quadrature and Differential Equations, SIAM, Philadelphia, Pa, USA, 1992.

[31] F. Stenger, "Numerical methods based on Whittaker cardinal, or Sinc functions," SIAM Review, vol. 23, no. 2, pp. 165-224, 1981.

[32] F. Stenger, Numerical Methods Based on Sinc and Analytic Functions, Springer, New York, NY, USA, 1993.

[33] R. P. Boas, Entire Functions, Academic Press, New York, NY, USA, 1954.

[34] M. S. P. Eastham, Theory of Ordinary Differential Operators, Van Nostrand Reinhold, London, UK, 1970.

[35] B. M. Levitan and I. S. Sargsjan, "Introduction to spectral theory: selfadjoint ordinary differential operators," in Translation of Mthematical Monographs, vol. 39, American Mathematical Society, Providence, RI, USA, 1975.

[36] M. H. Annaby and M. M. Tharwat, "On sampling theory and eigenvalue problems with an eigenparameter in the boundary conditions," SUT Journal of Mathematics, vol. 42, no. 2, pp. 157176, 2006.

[37] M. M. Tharwat, "Discontinuous Sturm-Liouville problems and associated sampling theories," Abstract and Applied Analysis, vol. 2011, Article ID 610232, 30 pages, 2011.

[38] C. T. Fulton, "Two-point boundary value problems with eigenvalue parameter contained in the boundary conditions," 
Proceedings of the Royal Society of Edinburgh A, vol. 77, no. 3-4, pp. 293-308, 1977.

[39] E. C. Titchmarsh, Eigenfunction Expansions Associated with Second-Order Differential Equations, Part 1, Clarendon Press, Oxford, UK, 1962.

[40] J. Walter, "Regular eigenvalue problems with eigenvalue parameter in the boundary condition," Mathematische Zeitschrift, vol. 133, pp. 301-312, 1973.

[41] M. H. Annaby and M. M. Tharwat, "The Hermite interpolation approach for computing eigenvalues of dirac systems," Mathematical and Computer Modelling, vol. 57, pp. 2459-2472, 2013.

[42] M. M. Tharwat, "Computing eigenvalues and Hermite interpolation for Dirac systems with eigenparameter in boundary conditions," Boundary Value Problems, vol. 2013, p. 36, 2013.

[43] M. M. Tharwat and A. H. Bhrawy, "Computation of eigenvalues of discontinuous Dirac system using Hermite interpolation technique," Advances in Difference Equations, vol. 2012, p. 59, 2012.

[44] K. Chadan and P. C. Sabatier, Inverse Problems in Quantum Scattering Theory, Springer, New York, NY, USA, 2nd edition, 1989.

[45] S. M. Al-Harbi, "Computing of eigenvalues of Sturm-Liouville problems with eigenparameter dependent boundary conditions," Iranian Journal of Science and Technology A, vol. 35, no. 4, pp. 293-299, 2011. 


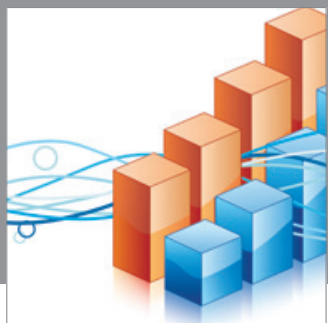

Advances in

Operations Research

mansans

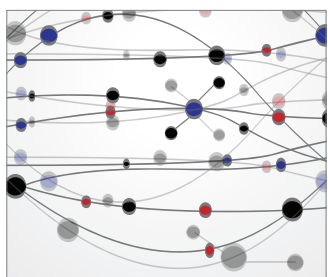

The Scientific World Journal
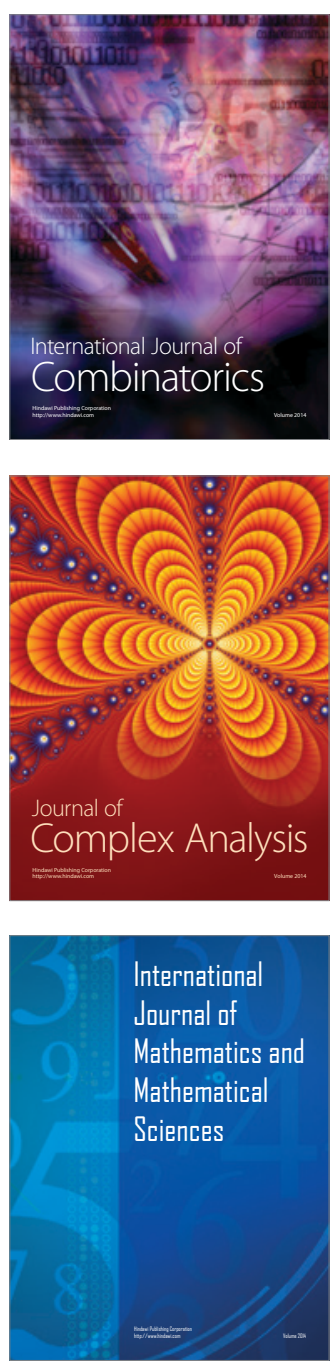
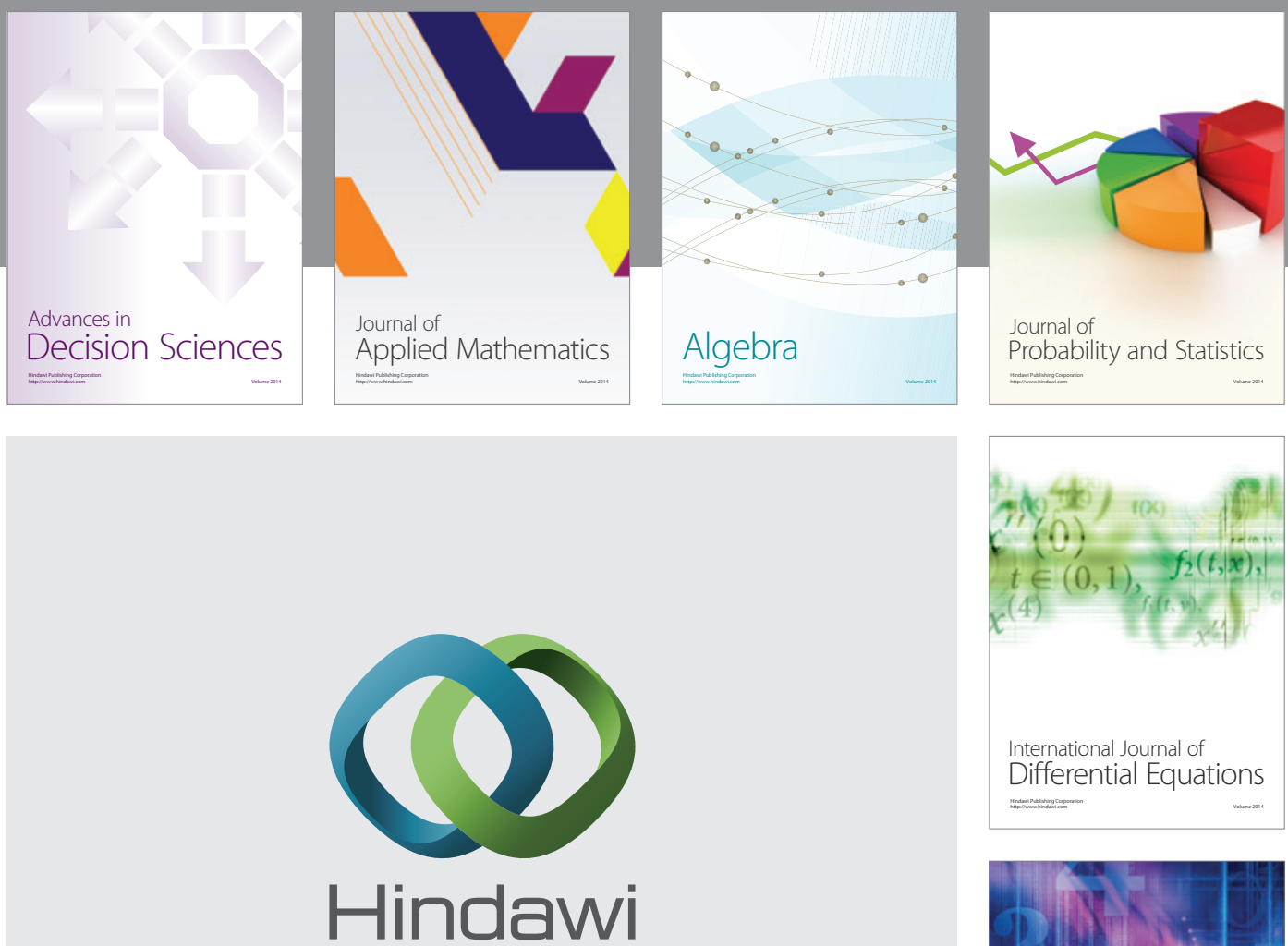

Submit your manuscripts at http://www.hindawi.com
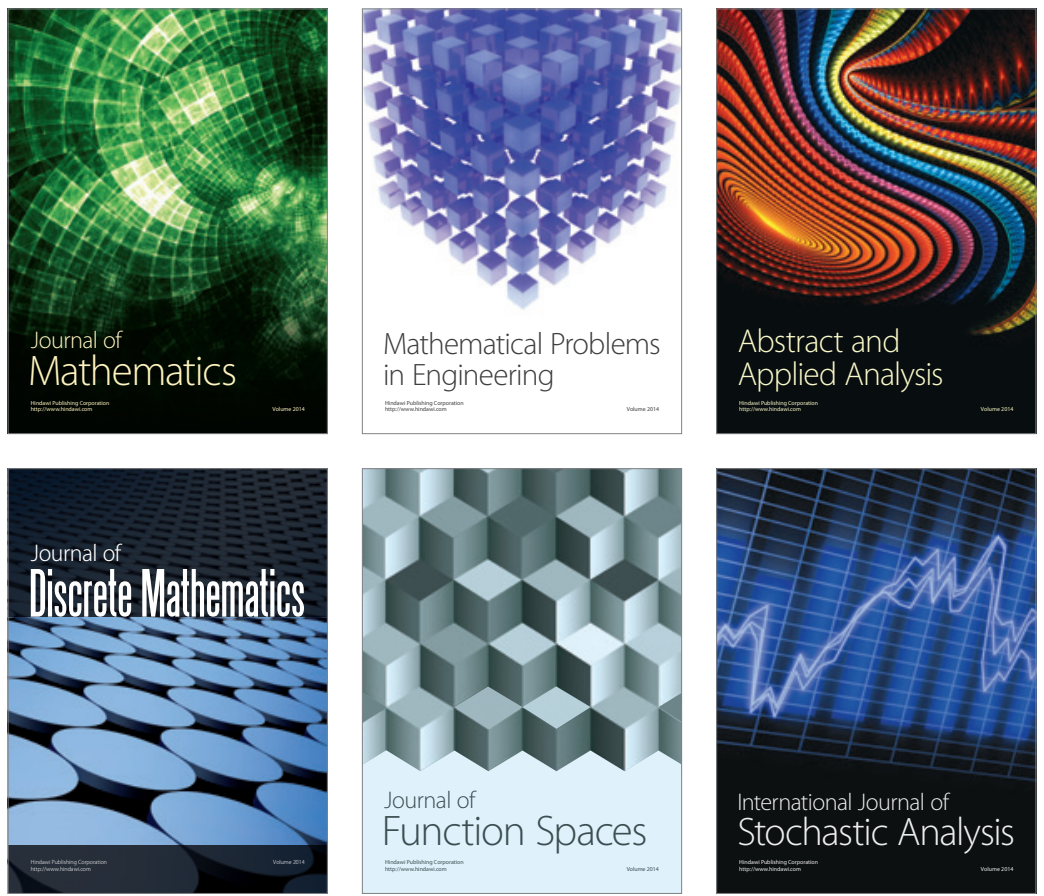

Journal of

Function Spaces

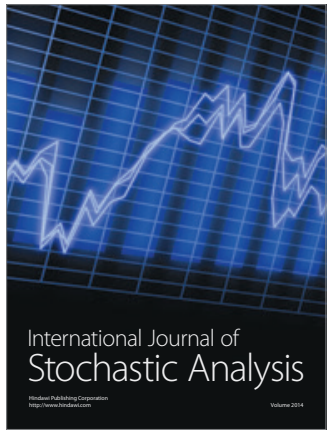

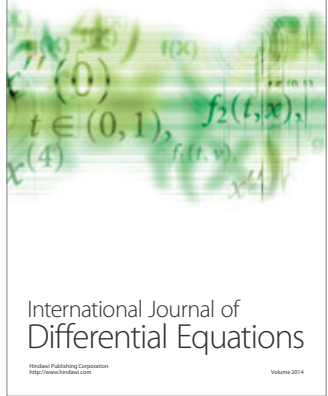
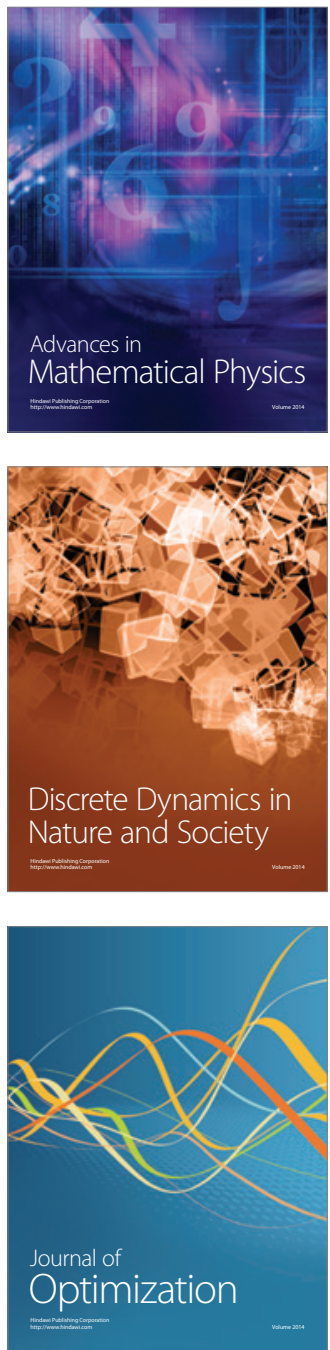